Netional Bjureau of Standards

Iibrary, N.W. Bldz

OCT 71965

\title{
Al1102 155102
}

Central Radio Propagation Laboratory TK6570

B7 147

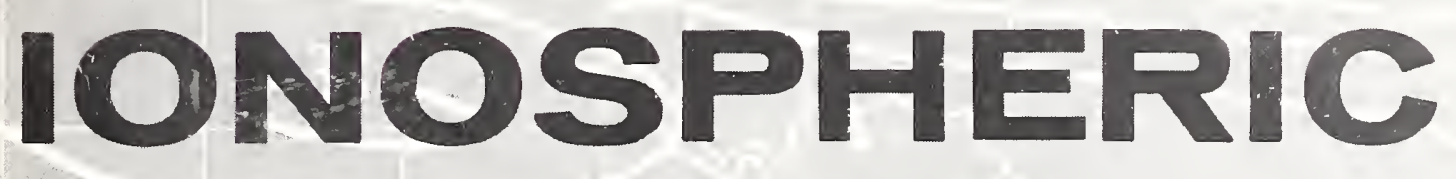

PREDICTIONS

\section{for}

December

1965
IMPORTANT

NOTICE

SEE

INTRODUCTION PAGE

U.S. DEPARTMENT of COMMERCE

National Bureau of Standards

Number 33/Issued September 1965 
Central Radio Propagation Laboratory

\section{Ionospheric Predictions}

Number 33

Issued

September 1965
The CRPL Ionospheric Predictions are issued monthly as an aid in determining the best sky-wave frequencies over any transmission path, at any time of day, for average conditions for the month. Issued three months in advance, each issue provides tables of numerical coefficients that define the functions describing the predicted worldwide distribution of fo F2 and $M(3000) F 2$ and maps for each even hour of universal time of MUF(Zero) F2 and MUF(4000) F2.

NoTE: Department of Defense personnel see back cover.

Use of funds for printing this publicntion approved by the Director of the Bureau of the Budget (June 19. 1961).

For sale by the Superintendent of Documents, U.S. Government Printing Office, Washington. D.C.. 20402. Price 25 cents.

Annual subscription ( 12 issues) $\$ 2.50$ (75 cents additional for foreign mailing).

\section{National Bureau of Standards}

The National Bureau of Standards serves as a principal focal point within the Federal Government for assuring maximum application of the physical and engineering sciences to the advancement of technology in industry and commerce. The Bureau is organized into four institutes as follows: The Institute for Basic Standarils provides the central basis within the United States for a complete and consistent system of physical measurement; its responsibilities include administration of the National Standard Reference Data System. The Institute for Materials Research conducts a broad range of plograms to plovide a better understanding of the basic properties and hehavior of materials and to make available reliable quantitative lata on their performance; it distributes a wide variety of carefully characterized reference materials to science and industry. The Institute for Applied Technology develops criteria for the evaluation of the performance of technological products and services, provides specialized information services to meet the needs of industry, and studies problems of technological innovation. The fourth institute, the Central Radio Propagation Laboratory, is described below.

\section{The Central Radio Propagation Laboratory}

The Central Radio Propagation Laboratory is the central agency of the Federal Government for obtaining and disseminating information on the propagation of electromagnetic waves, on the electromagnetic properties of man's environment, on the nature of electromagnetic noise and interference, and on methods for the more efficient use of the electromagnetic spectrum for telecommunication purposes. In carrying out these responsibilities, the Central Radio Propagation Laboratory:

1. Acts as the primary agency of the Federal Government for the conduct of basic and applied research in these fields;
2. Acts as the central repository fol data, l'eports, and information in these fields;

3. Furnishes advisory and consultative services in these fields to industry and to other government and non-government organizations;

4. Performs scientific tiaison with other countries to advance knowledge in these field's, including that liaison required by international responsibilities and agreements;

5. Prepares and issues predictions of electromagnetic wave propagation conditions, and warnings of disturbances in these conditions. 


\section{NOTICE}

\section{TRIAL PERIOD FOR POLAR MAPS EXTENDED}

The trial period for the polar prediction maps has been extended six months in order to permit more time to evaluate their utility. Some favorable comnents have been received but the response has been insufficient to justify the expense and effort required to publish them. Therefore, please send your comments as soon as possible if you wish these to continue.

\section{ANNOUNCEMENT OF PUBLICATION OF NBS MONOGRAPH 80 \\ IONOSPHERIC RADIO PROPAGATION}

By Kenneth Davies of the Central Radio Propagation Laboratory, Boulder, Colorado.

This new textbook provides an authoritative and comprehensive digest of current information on the ionosphere. Emphasis is placed on the physics of the ionosphere and theoretical developments required for an understanding of ionospheric radio propagation.

Orders, accompanied by $\$ 2.75$ remittance, should be sent to: Superintendent of Documents, U.S. Government Printing Office, Washington, D.C., 20402.

\section{INTRODUCTION}

Tables 1 and 2 , presenting predicted coefficients defining the numerical map functions for the worldwide variation of foF 2 and $\mathrm{M}(3000) \mathrm{F} 2$, provide the basic prediction or F2-layer propagation. With additional auxiliary information, these coefficients may be used as input data for electronic computer programs solving specific high-frequency propagation problems. The graphical maps, which are derived from the basic predictions, are provided for those unable to make use of an electronic computer. Instructions for use of these maps, figures 1 through 24, may be found in National Bureau of Standards Handbook 90, "Handbook for CRPL Ionospheric Predictions Based on Numerical Methods of Mapping," which also includes required additional data, nomographs and graphical aids, and may be purchased from the Superintendent of Documents, U.S. Government Printing Office, Washington D.C., 20402, price 40 cents. The predicted sunspot number used for this month is shown in table $A$, which also lists previous observed and predicted Zurich smoothed relative sunspot numbers. Figure A shows the recent trend of solar activity, with both predicted and observed Zurich smoothed relative sunspot numbers.

The basic numerical mapping equations, their interpretation, and methods of using numerical maps are described in papers by W. B. Jones and R. M Gallet, "The Representation of Diurnal and Geographic Variations of Ionospheric Data by Numerical Mapping," vol. $66 \mathrm{D}$, No. 4, July-Aug. 1962, pages 419-438, and "Methods for Applying Numerical Maps of Ionospheric Characteristies," vol. 66D, No. 6, Nov.-Dec. 1962, pages 649-662, both in the Journal of Research of the National Bureau of Standards, Section D. Radio Propagation. The predicted numerical map coefficients of tables 1 and 2 may be purchased in the form of a tested set of punched cards. Write to the Prediction Services Section, Central Radio Propagation Laboratory, National Bureau of Standards, Boulder, Colorado, 80301, to arrange for purchase of the punched cards, and for information and advice on the application of computer methods and numerical prediction maps to specific propagation problems.

Members of the U.S. Army, Navy, or Air Force desiring Handbook 90 and the monthly issues of Ionospheric Prediction should send requests to the proper service address; for Navy: The Director, Naval Communications, Department of the Navy, Washington, D.C., 20350; for Air Force: Directorate of Command Control and Communications, Headquarters, United States Air Force, Washington, D.C., 20330. ATTN: AFOCCAA. Army personnel should requisition these through normal publication channels, and should refer to Handbook 90 as TM 11-499 and to the monthly ionospheric predictions as TB $11-499-($ ), with the serial number of the desired monthly predictions booklet inserted in the parenthesis. (For exampie, for Ionospheric Predictions Number 33, issued September 1965 and containing predictions for December 1965, the Army number would be TB 11-499-(33).)

Information on the physics of the ionosphere and the theory of radio wave propagation, including such problems as absorption, field intensity, etc., may be found in National Bureau of Standards Monograph 80, "Ionospheric Radio Propagation," by Kenneth Davies, which may be purchased from the Superintendent of Documents, U.S. Government Printing Office, Washington, D.C., 20402, price $\$ 2.75$. Additional information on radio noise may be found in C.C.I.R. Report Number 322, "Revision of Atmospheric Noise Data," International Telecommunications Union, Geneva, 1964.

Reports to this Laboratory of experience with these predictions would be appreciated. Correspondence should be addressed to the Prediction Services Section, Central Radio Propagation Laboratory, National Bureau of Standards, Boulder, Colorado, 80301. 
$\underline{\text { Table A }}$

Observed and Predicted Zurich Snoothed Relative Sunspot Numbers

\begin{tabular}{|c|c|c|c|c|c|c|c|c|c|c|c|c|}
\hline Month & Jan. & Feb. & Mar. & Apr. & May & June & July & Aug. & Sept. & - Oct. & Nov. & Dec. \\
\hline 1954 & $\begin{array}{c}6 \\
(14)\end{array}$ & $\begin{array}{c}6 \\
(12)\end{array}$ & $\begin{array}{c}4 \\
(11)\end{array}$ & $\begin{array}{c}3 \\
(10)\end{array}$ & $\begin{array}{c}4 \\
(10)\end{array}$ & $\begin{array}{c}4 \\
(9)\end{array}$ & $\begin{array}{c}5 \\
(8)\end{array}$ & $\begin{array}{c}7 \\
(8)\end{array}$ & $\begin{array}{c}8 \\
(8)\end{array}$ & $\begin{array}{c}8 \\
(10)\end{array}$ & $\begin{array}{c}10 \\
(10)\end{array}$ & $\begin{array}{c}12 \\
(11)\end{array}$ \\
\hline 1955 & $\begin{array}{c}14 \\
(12)\end{array}$ & $\begin{array}{c}16 \\
(14)\end{array}$ & $\begin{array}{c}20 \\
(14)\end{array}$ & $\begin{array}{c}23 \\
(13)\end{array}$ & $\begin{array}{c}29 \\
(16)\end{array}$ & $\begin{array}{c}35 \\
(18)\end{array}$ & $\begin{array}{c}40 \\
(22)\end{array}$ & $\begin{array}{c}46 \\
(27)\end{array}$ & $\begin{array}{c}55 \\
(30)\end{array}$ & $\begin{array}{c}64 \\
(31)\end{array}$ & $\begin{array}{c}73 \\
(35)\end{array}$ & $\begin{array}{c}81 \\
(42)\end{array}$ \\
\hline 1956 & $\begin{array}{c}89 \\
(48)\end{array}$ & $\begin{array}{c}98 \\
(53)\end{array}$ & $\begin{array}{l}109 \\
(60)\end{array}$ & $\begin{array}{l}119 \\
(68)\end{array}$ & $\begin{array}{l}127 \\
(77)\end{array}$ & $\begin{array}{l}137 \\
(89)\end{array}$ & $\begin{array}{l}146 \\
(95)\end{array}$ & $\begin{array}{c}150 \\
(105)\end{array}$ & $\begin{array}{c}151 \\
(119)\end{array}$ & $\begin{array}{c}156 \\
(135)\end{array}$ & $\begin{array}{c}160 \\
(147)\end{array}$ & $\begin{array}{c}164 \\
(150)\end{array}$ \\
\hline 1957 & $\begin{array}{c}170 \\
(150)\end{array}$ & $\begin{array}{c}172 \\
(150)\end{array}$ & $\begin{array}{c}174 \\
(150)\end{array}$ & $\begin{array}{c}181 \\
(150)\end{array}$ & $\begin{array}{c}186 \\
(150)\end{array}$ & $\begin{array}{c}188 \\
(150)\end{array}$ & $\begin{array}{c}191 \\
(150)\end{array}$ & $\begin{array}{c}194 \\
(150)\end{array}$ & $\begin{array}{c}197 \\
(150)\end{array}$ & $\begin{array}{c}200 \\
(150)\end{array}$ & $\begin{array}{c}201 \\
(150)\end{array}$ & $\begin{array}{c}200 \\
(150)\end{array}$ \\
\hline 1958 & $\begin{array}{c}199 \\
(150)\end{array}$ & $\begin{array}{c}201 \\
(150)\end{array}$ & $\begin{array}{c}201 \\
(150)\end{array}$ & $\begin{array}{c}197 \\
(150)\end{array}$ & $\begin{array}{c}191 \\
(150)\end{array}$ & $\begin{array}{c}187 \\
(150)\end{array}$ & $\begin{array}{c}185 \\
(150)\end{array}$ & $\begin{array}{c}185 \\
(150)\end{array}$ & $\begin{array}{c}184 \\
(150)\end{array}$ & $\begin{array}{c}182 \\
(150)\end{array}$ & $\begin{array}{c}181 \\
(150)\end{array}$ & $\begin{array}{c}180 \\
(150)\end{array}$ \\
\hline 1959 & $\begin{array}{c}179 \\
(150)\end{array}$ & $\begin{array}{c}177 \\
(150)\end{array}$ & $\begin{array}{c}174 \\
(150)\end{array}$ & $\begin{array}{c}169 \\
(150)\end{array}$ & $\begin{array}{c}165 \\
(146)\end{array}$ & $\begin{array}{c}161 \\
(143)\end{array}$ & $\begin{array}{l}156 \\
(141)\end{array}$ & $\begin{array}{c}151 \\
(142)\end{array}$ & $\begin{array}{c}146 \\
(141)\end{array}$ & $\begin{array}{c}141 \\
(139)\end{array}$ & $\begin{array}{c}137 \\
(137)\end{array}$ & $\begin{array}{c}132 \\
(137)\end{array}$ \\
\hline 1960 & $\begin{array}{c}129 \\
(136)\end{array}$ & $\begin{array}{c}125 \\
(135)\end{array}$ & $\begin{array}{c}122 \\
(133)\end{array}$ & $\begin{array}{c}120 \\
(130)\end{array}$ & $\begin{array}{c}117 \\
(125)\end{array}$ & $\begin{array}{c}114 \\
(120)\end{array}$ & $\begin{array}{c}109 \\
(118)\end{array}$ & $\begin{array}{c}102 \\
(115)\end{array}$ & $\begin{array}{c}98 \\
(110)\end{array}$ & $\begin{array}{c}93 \\
(108)\end{array}$ & $\begin{array}{c}88 \\
(105)\end{array}$ & $\begin{array}{c}84 \\
(100)\end{array}$ \\
\hline 1961 & $\begin{array}{c}80 \\
(100)\end{array}$ & $\begin{array}{c}75 \\
(90)\end{array}$ & $\begin{array}{c}69 \\
(90)\end{array}$ & $\begin{array}{c}64 \\
(90)\end{array}$ & $\begin{array}{c}60 \\
(85)\end{array}$ & $\begin{array}{c}56 \\
(85)\end{array}$ & $\begin{array}{c}53 \\
(80)\end{array}$ & $\begin{array}{c}52 \\
(75)\end{array}$ & $\begin{array}{c}52 \\
(70)\end{array}$ & $\begin{array}{c}51 \\
(70)\end{array}$ & $\begin{array}{c}50 \\
(65)\end{array}$ & $\begin{array}{c}49 \\
(60)\end{array}$ \\
\hline 1962 & $\begin{array}{c}45 \\
(60)\end{array}$ & $\begin{array}{c}42 \\
(50)\end{array}$ & $\begin{array}{c}40 \\
(48)\end{array}$ & $\begin{array}{c}39 \\
(45)\end{array}$ & $\begin{array}{c}39 \\
(42)\end{array}$ & $\begin{array}{c}38 \\
(37)\end{array}$ & $\begin{array}{c}37 \\
(34)\end{array}$ & $\begin{array}{c}35 \\
(31)\end{array}$ & $\begin{array}{c}33 \\
(29)\end{array}$ & $\begin{array}{c}31 \\
(28)\end{array}$ & $\begin{array}{c}30 \\
(27)\end{array}$ & $\begin{array}{c}30 \\
(34)\end{array}$ \\
\hline 1963 & $\begin{array}{c}29 \\
(31)\end{array}$ & $\begin{array}{c}30 \\
(28)\end{array}$ & $\begin{array}{c}30 \\
(26)\end{array}$ & $\begin{array}{c}29 \\
(25)\end{array}$ & $\begin{array}{c}29 \\
(25)\end{array}$ & $\begin{array}{c}28 \\
(25)\end{array}$ & $\begin{array}{c}28 \\
(23)\end{array}$ & $\begin{array}{c}27 \\
(21)\end{array}$ & $\begin{array}{c}27 \\
(20)\end{array}$ & $\begin{array}{c}26 \\
(18)\end{array}$ & $\begin{array}{c}24 \\
(18)\end{array}$ & $\begin{array}{c}21 \\
(17)\end{array}$ \\
\hline 1964 & $\begin{array}{c}20 \\
(17)\end{array}$ & $\begin{array}{c}18 \\
(17)\end{array}$ & $\begin{array}{c}15 \\
(17)\end{array}$ & $\begin{array}{c}13 \\
(17)\end{array}$ & $\begin{array}{c}11 \\
(17)\end{array}$ & $\begin{array}{c}10 \\
(17)\end{array}$ & $\begin{array}{c}10 \\
(17)\end{array}$ & $\begin{array}{c}10 \\
(17)\end{array}$ & $\begin{array}{c}10 \\
(17.5)\end{array}$ & $\begin{array}{c}10 \\
(1.7 .3)\end{array}$ & $\begin{array}{c}10 \\
(17.0)\end{array}$ & $\begin{array}{c}11 \\
(17.0)\end{array}$ \\
\hline 1966 & \multicolumn{6}{|c|}{$(15.0)(16.0)(16.0)(16.0)(15.0)(17.0)$} & \multicolumn{6}{|c|}{$(21.0)(28.9)(22.0)(22.0)(26.0)(29.0) *$} \\
\hline
\end{tabular}

Note: Final numbers are listed through June 1964, the succeeding values being based on provisional data. The predicted numbers are in parentheses.

* Number used for predictions in this issue. 


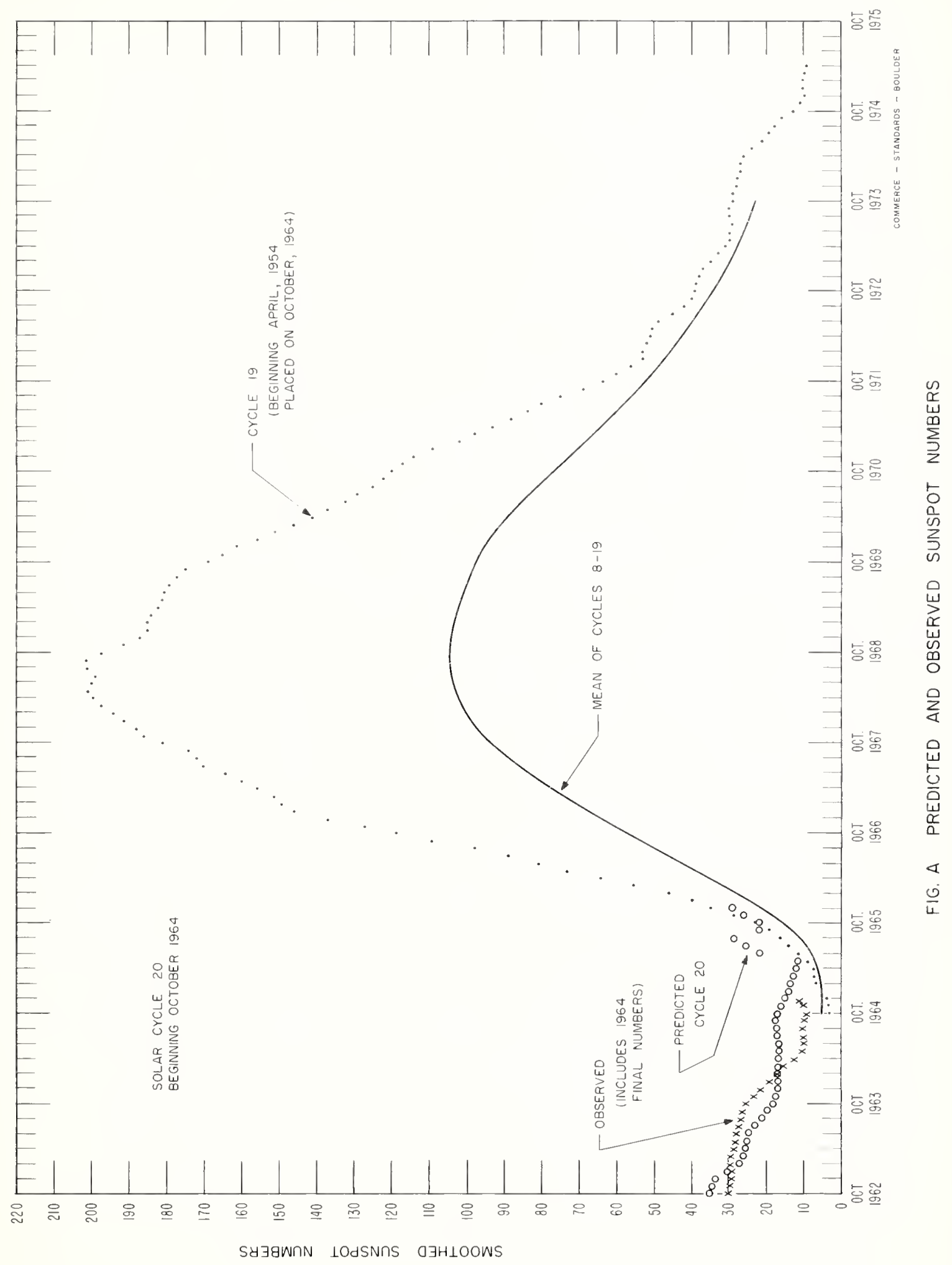




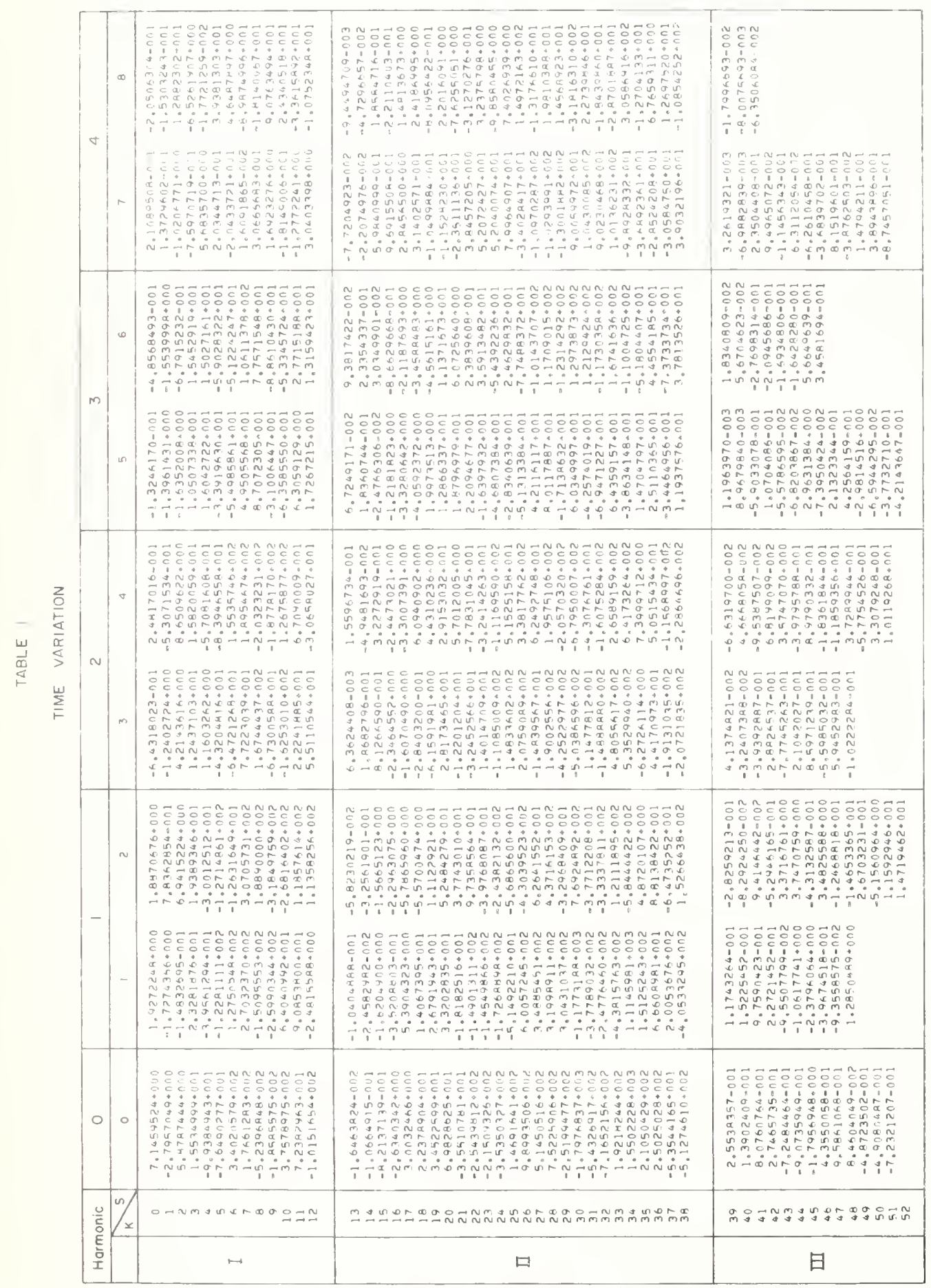

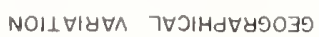

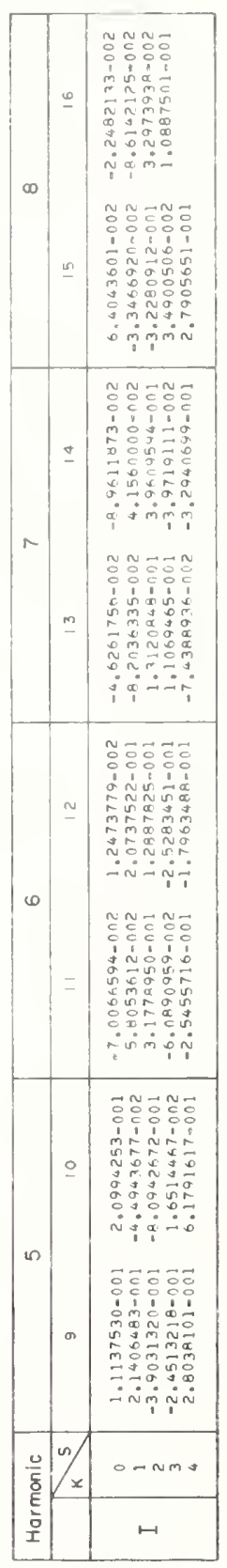

NOI $\perp \nabla I Y \forall A$ ᄀ७ग1Hd $\forall$ Y9O39 


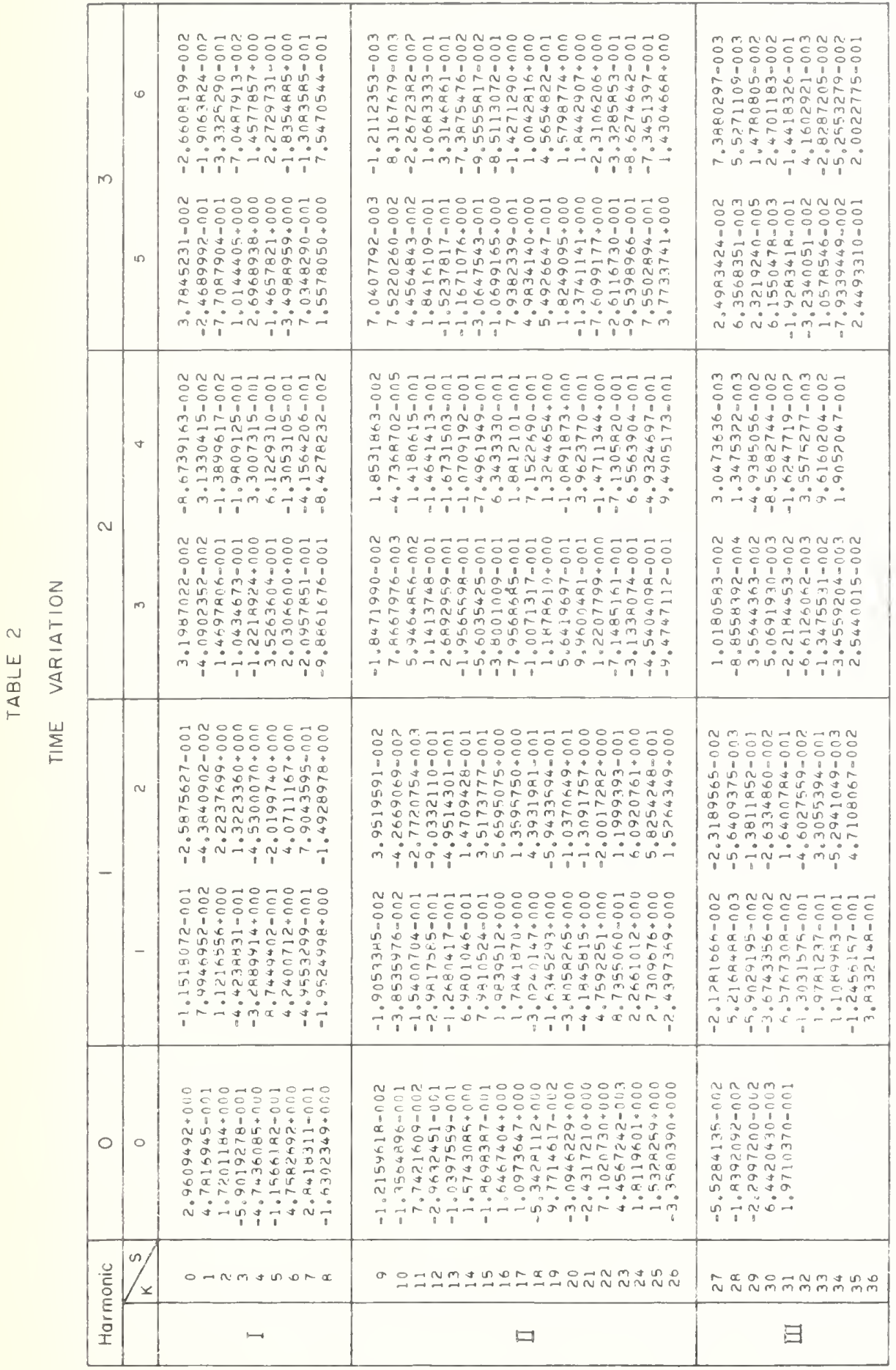

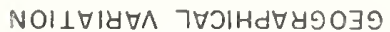

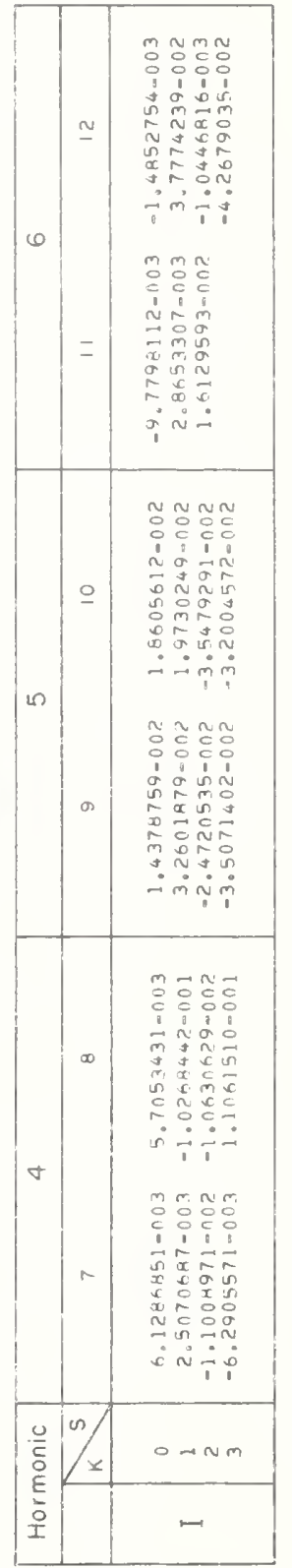

NOIL

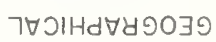



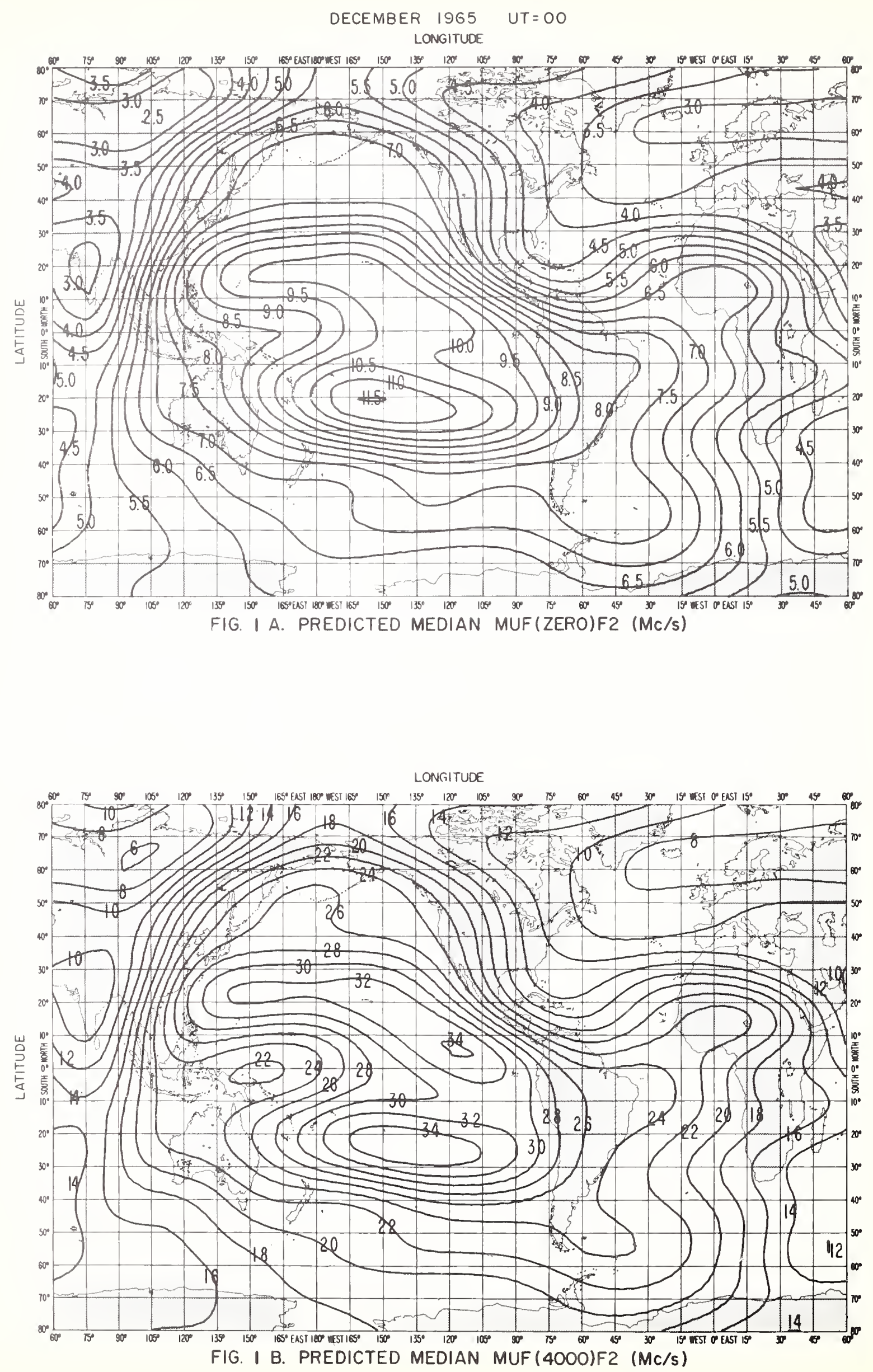


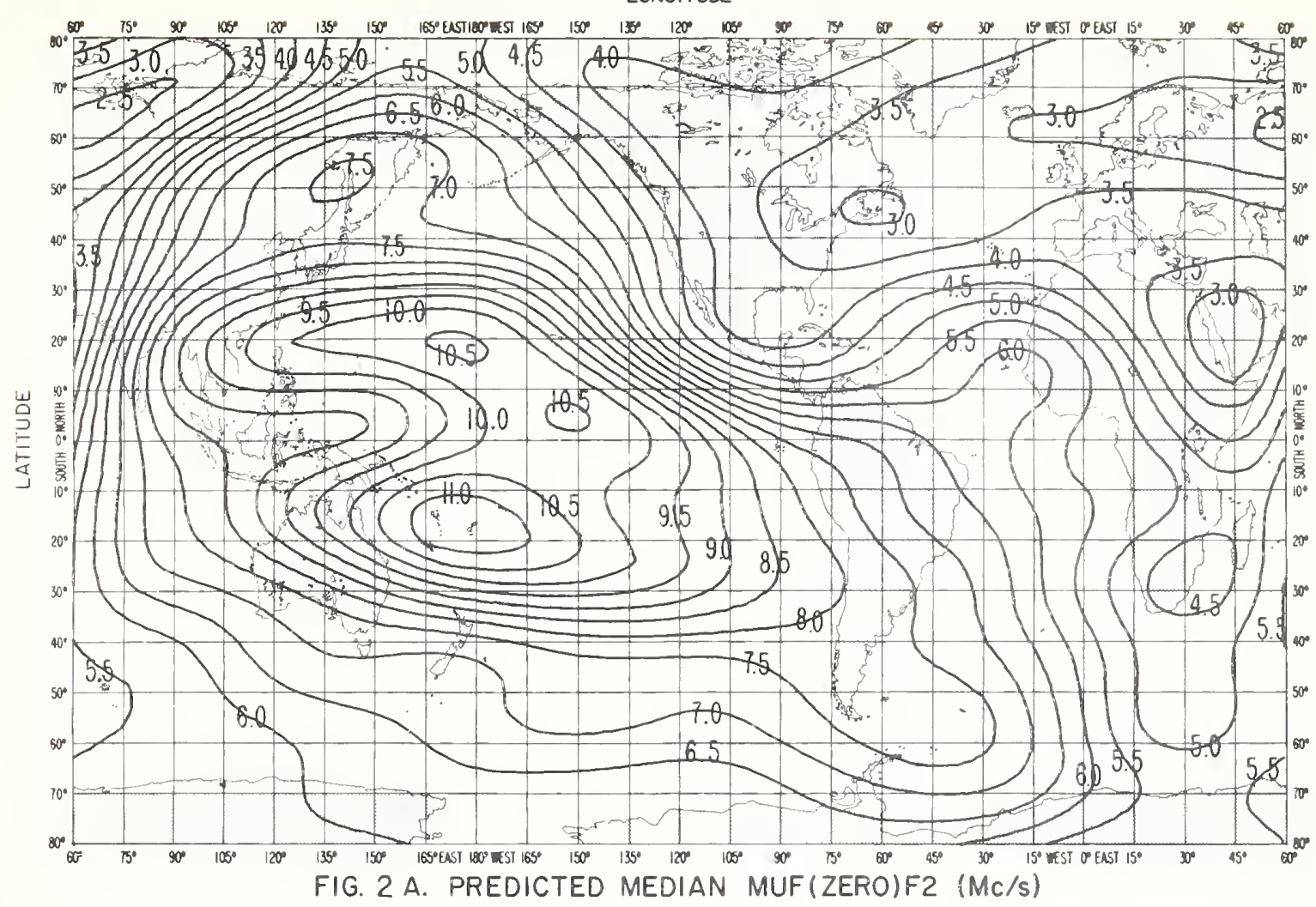

LONGITUDE

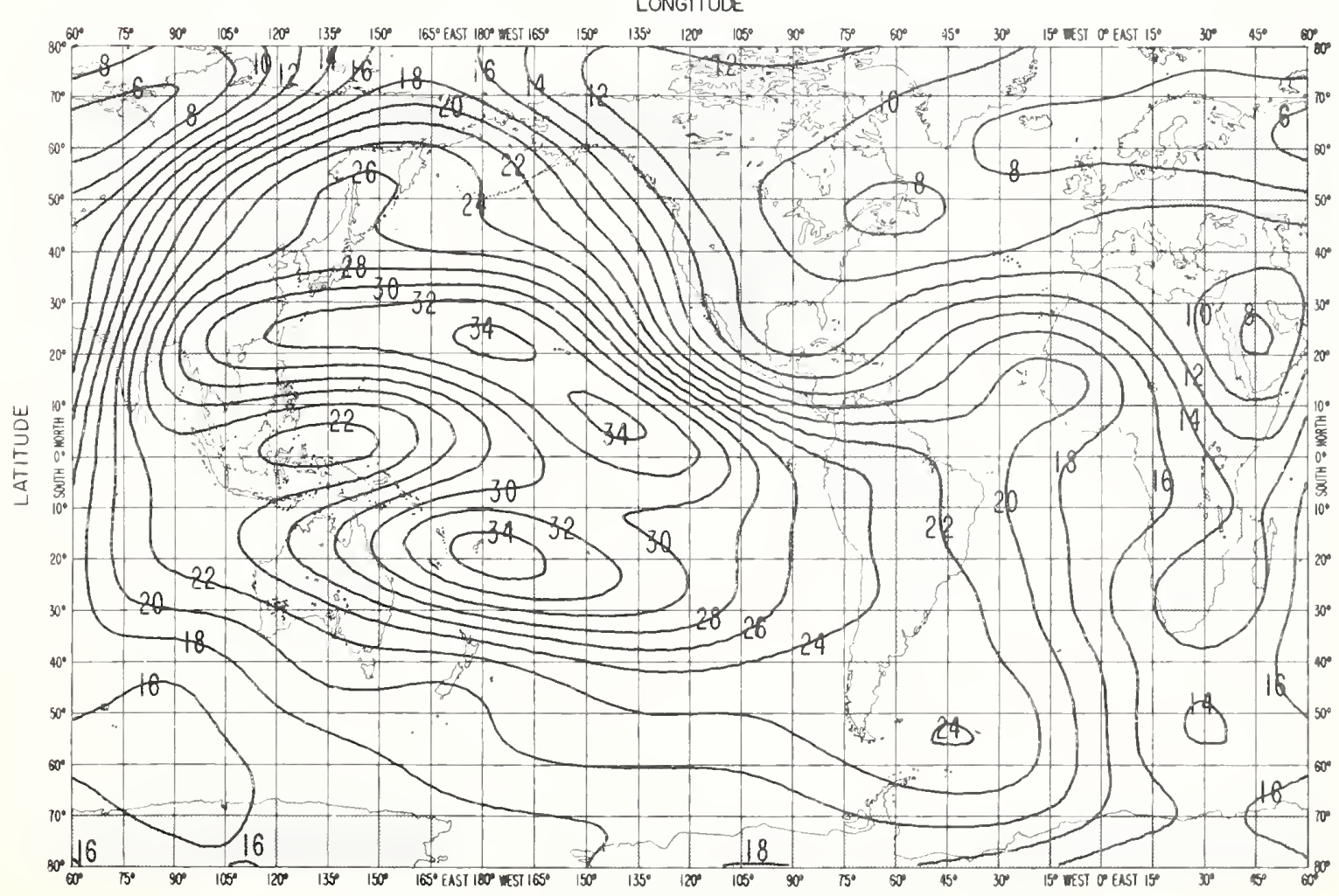

FIG. 2 B. PREDICTED MEDIAN MUF (4000)F2 (Mc/s) 


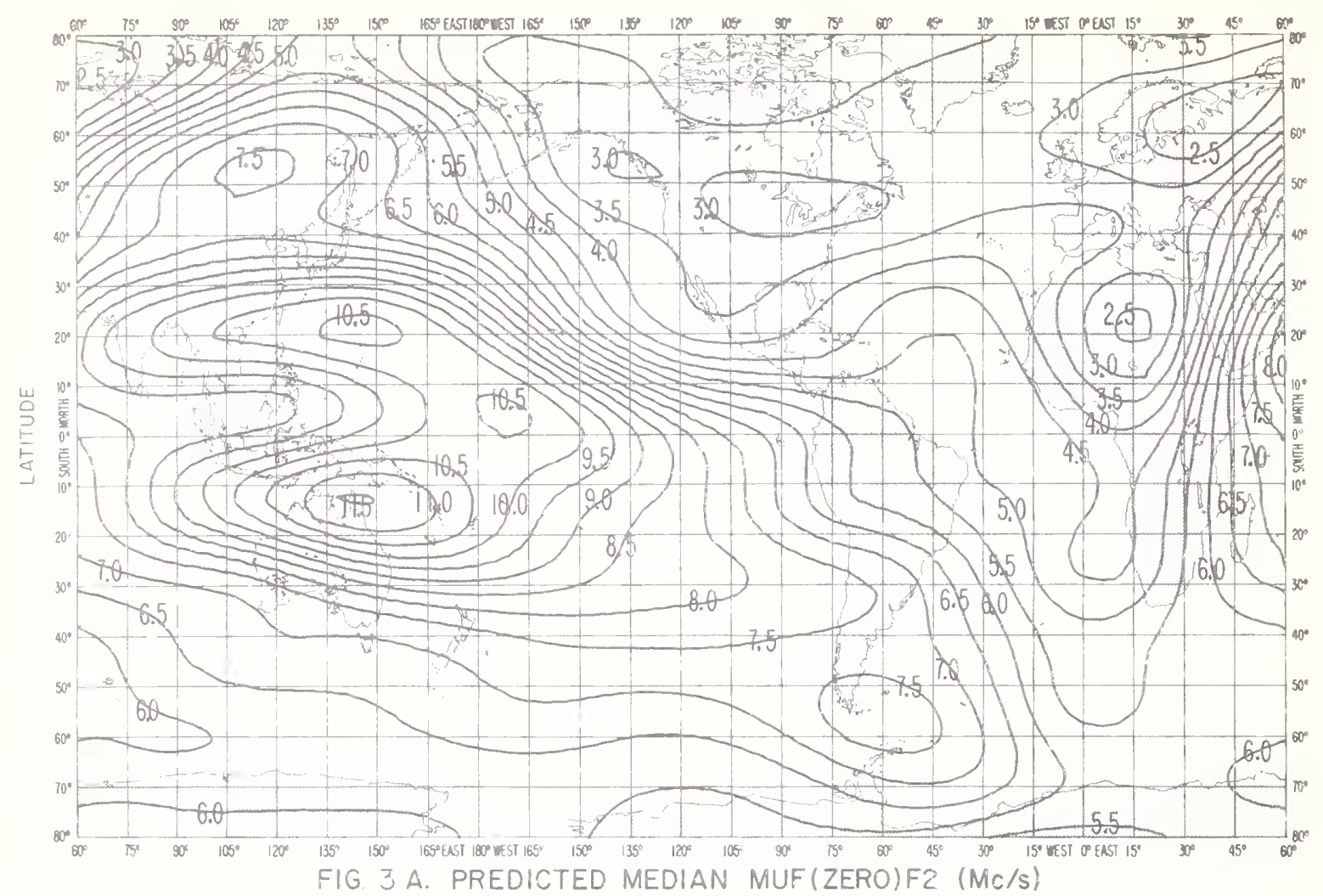

LONGITUDE

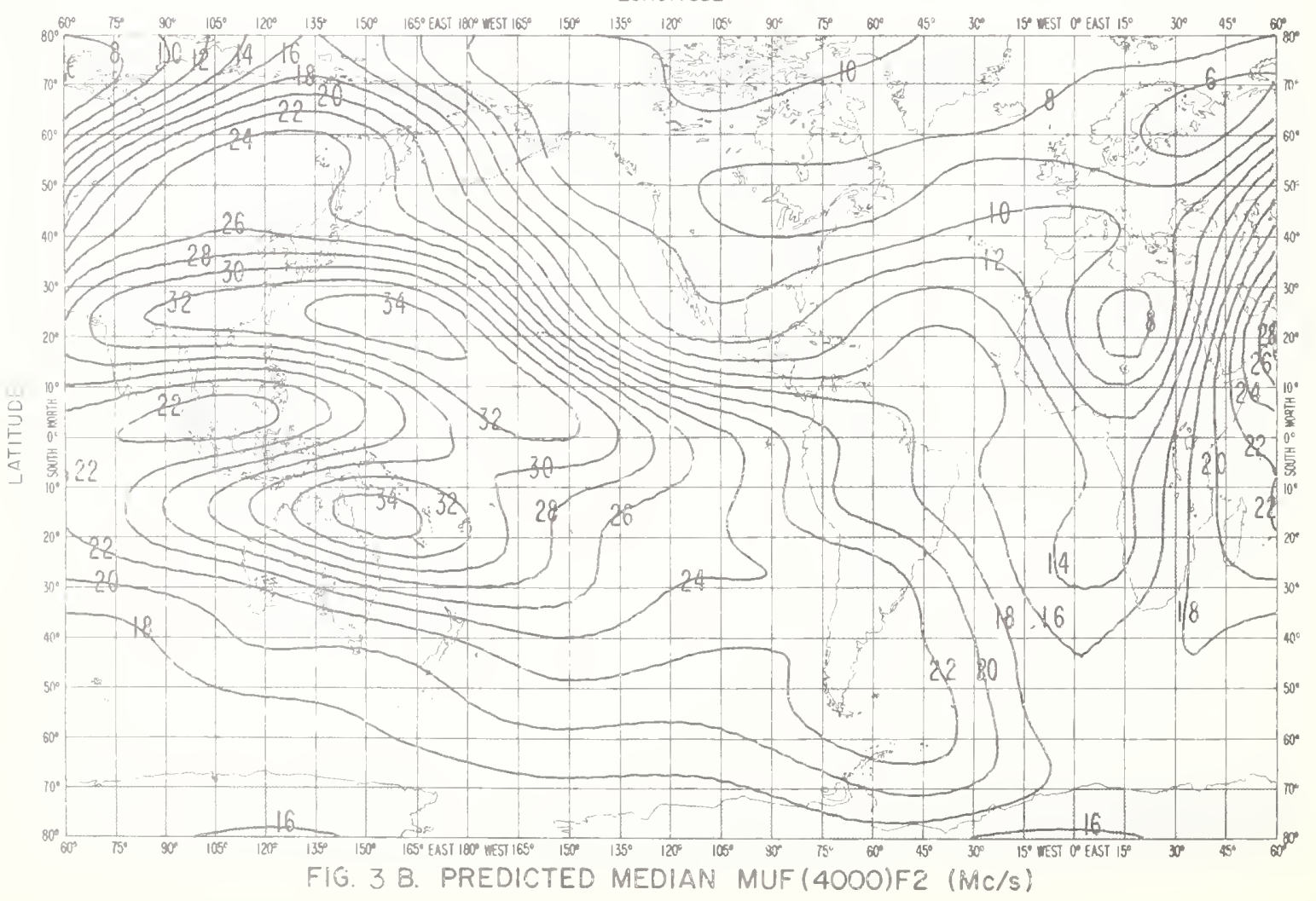




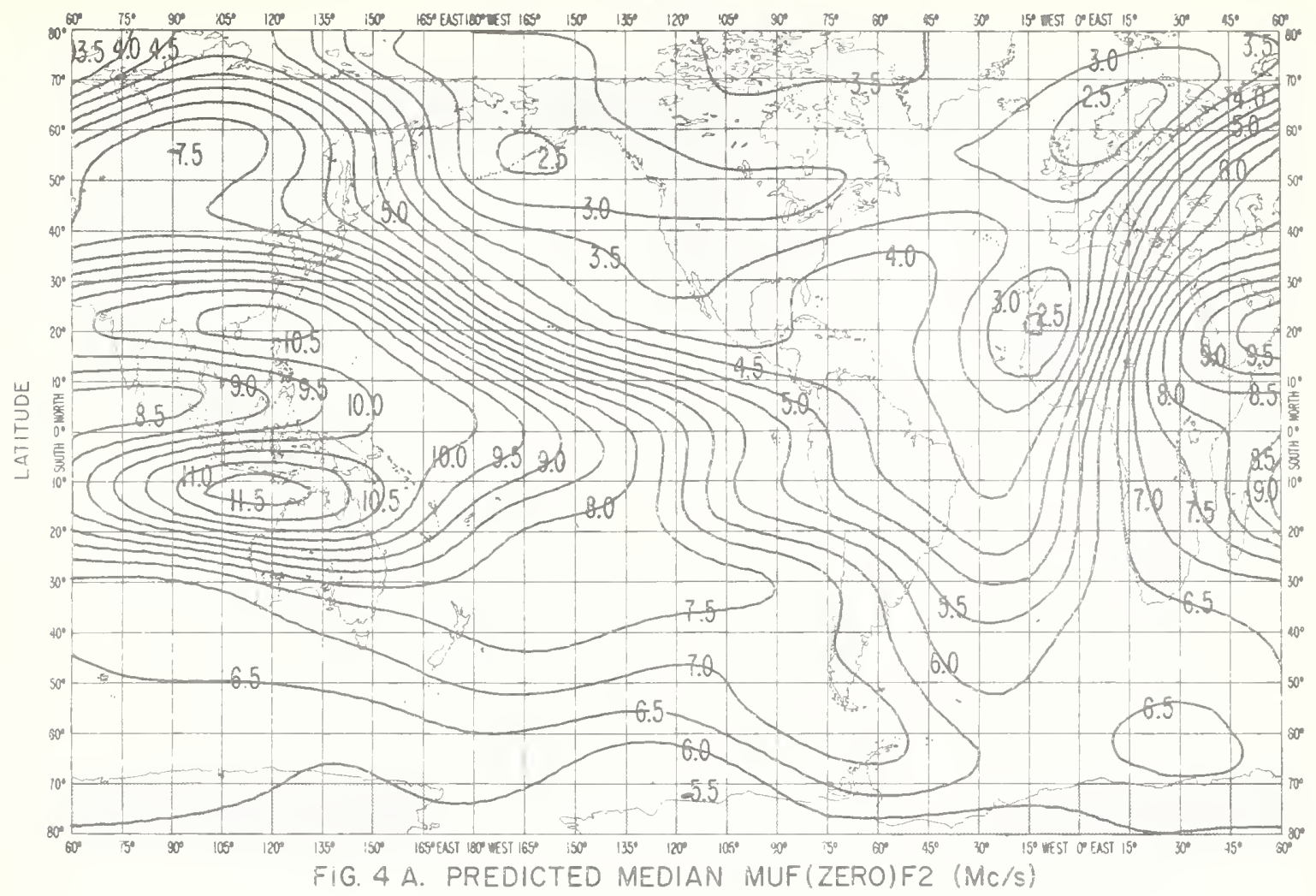

LONGITUDE

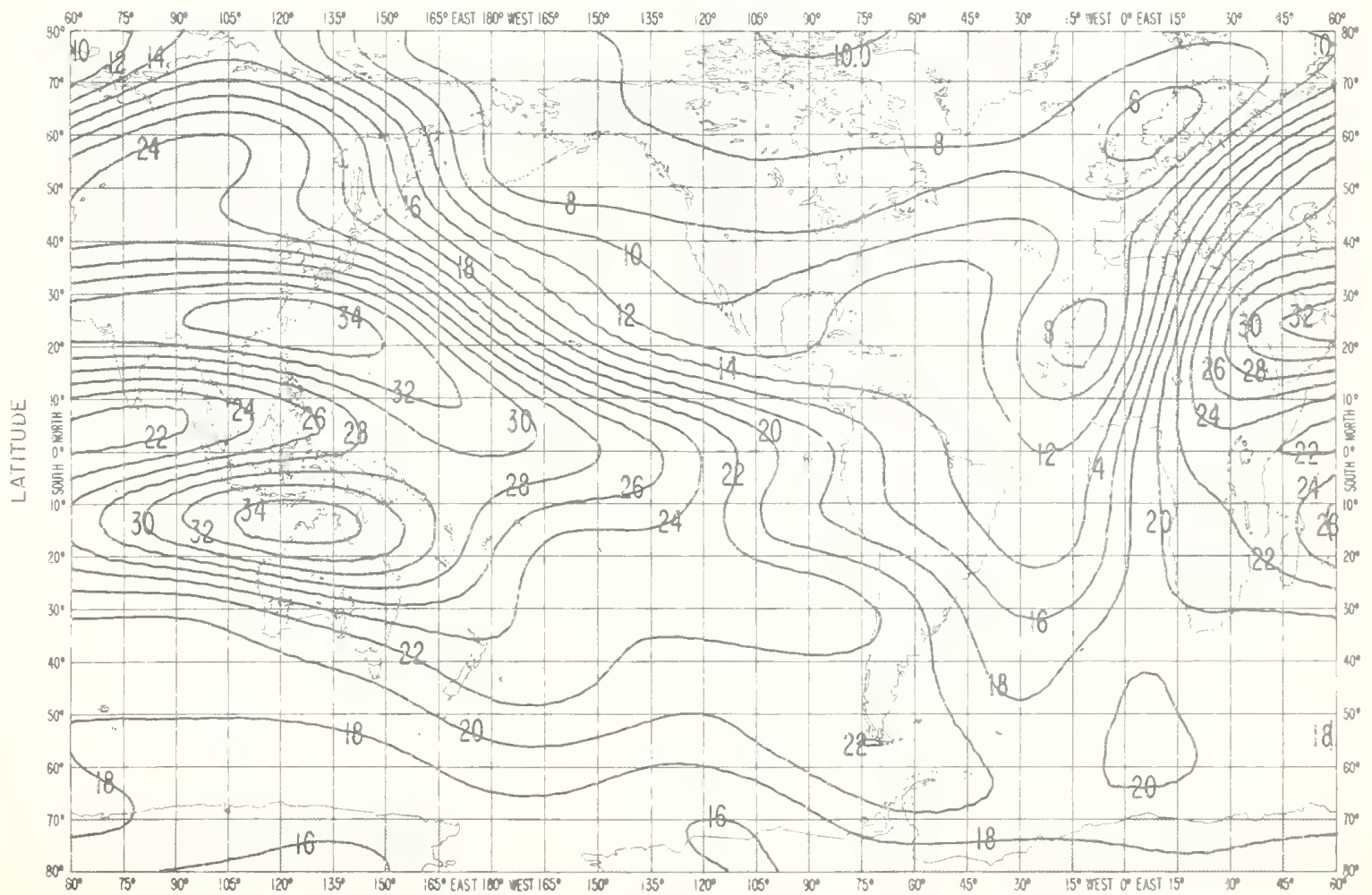

FIG. 4 B. PREDICTED MEDIAN MUF (4000)F2 ( $\mathrm{Mc} / \mathrm{s})$ 


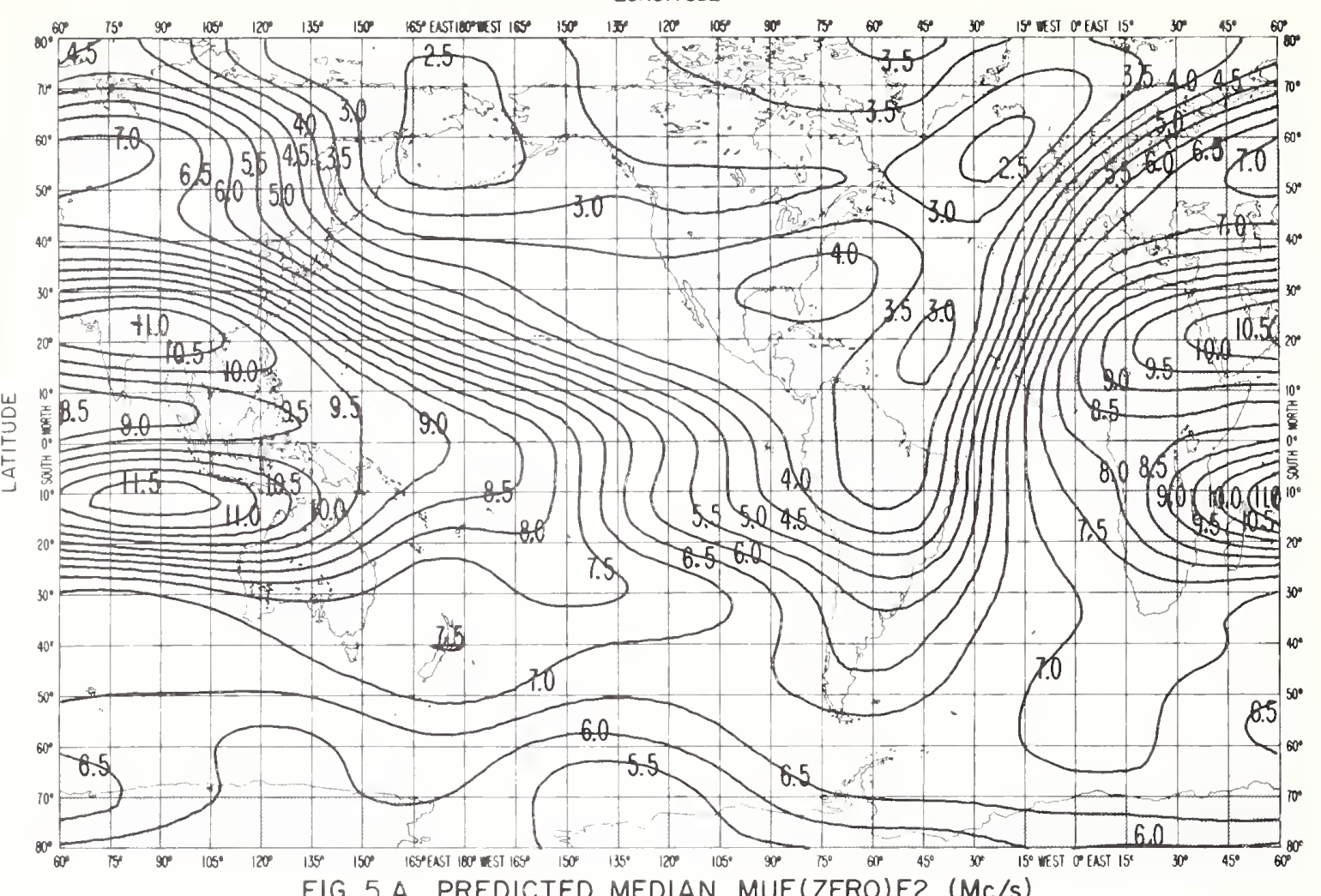

LONGITUDE

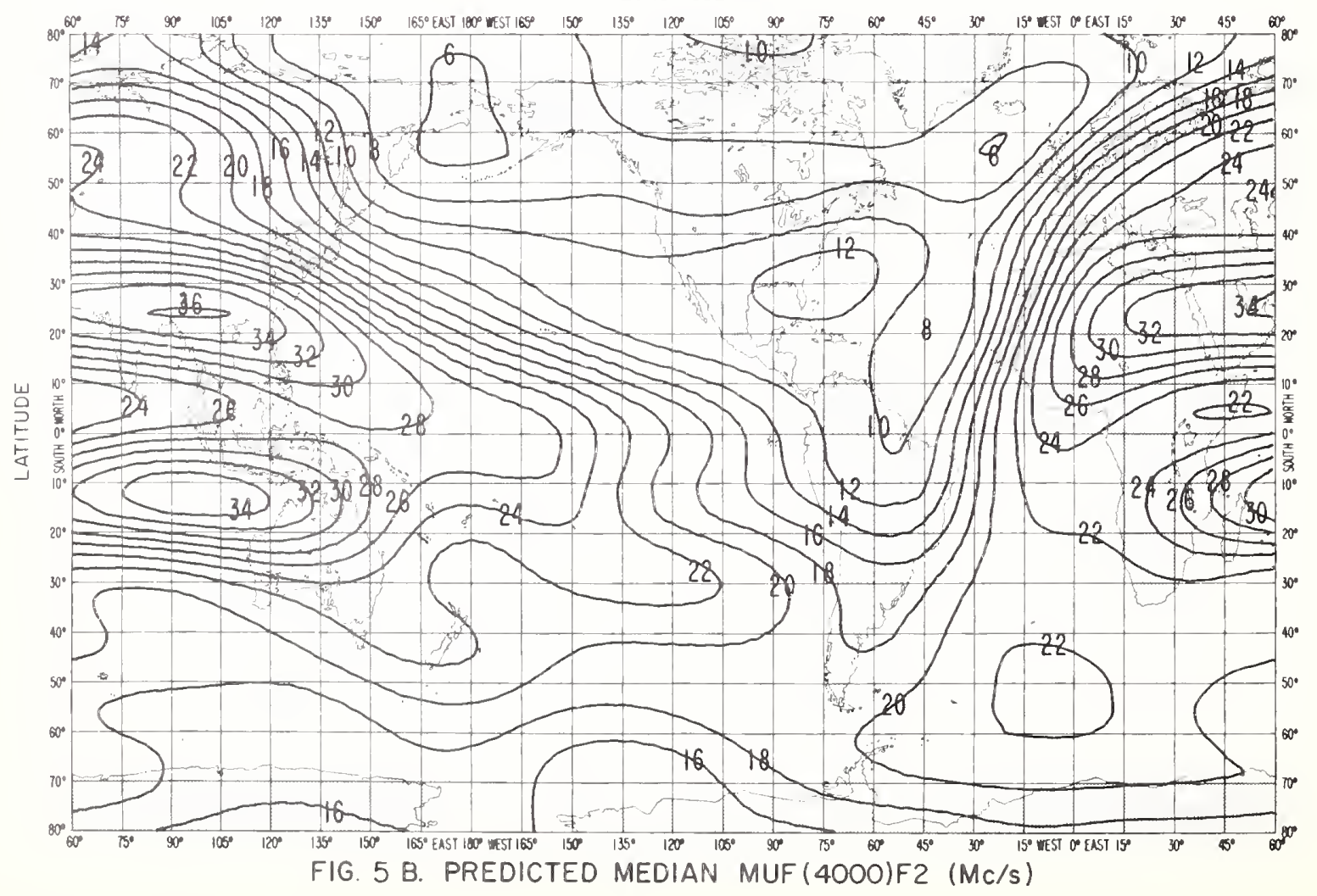



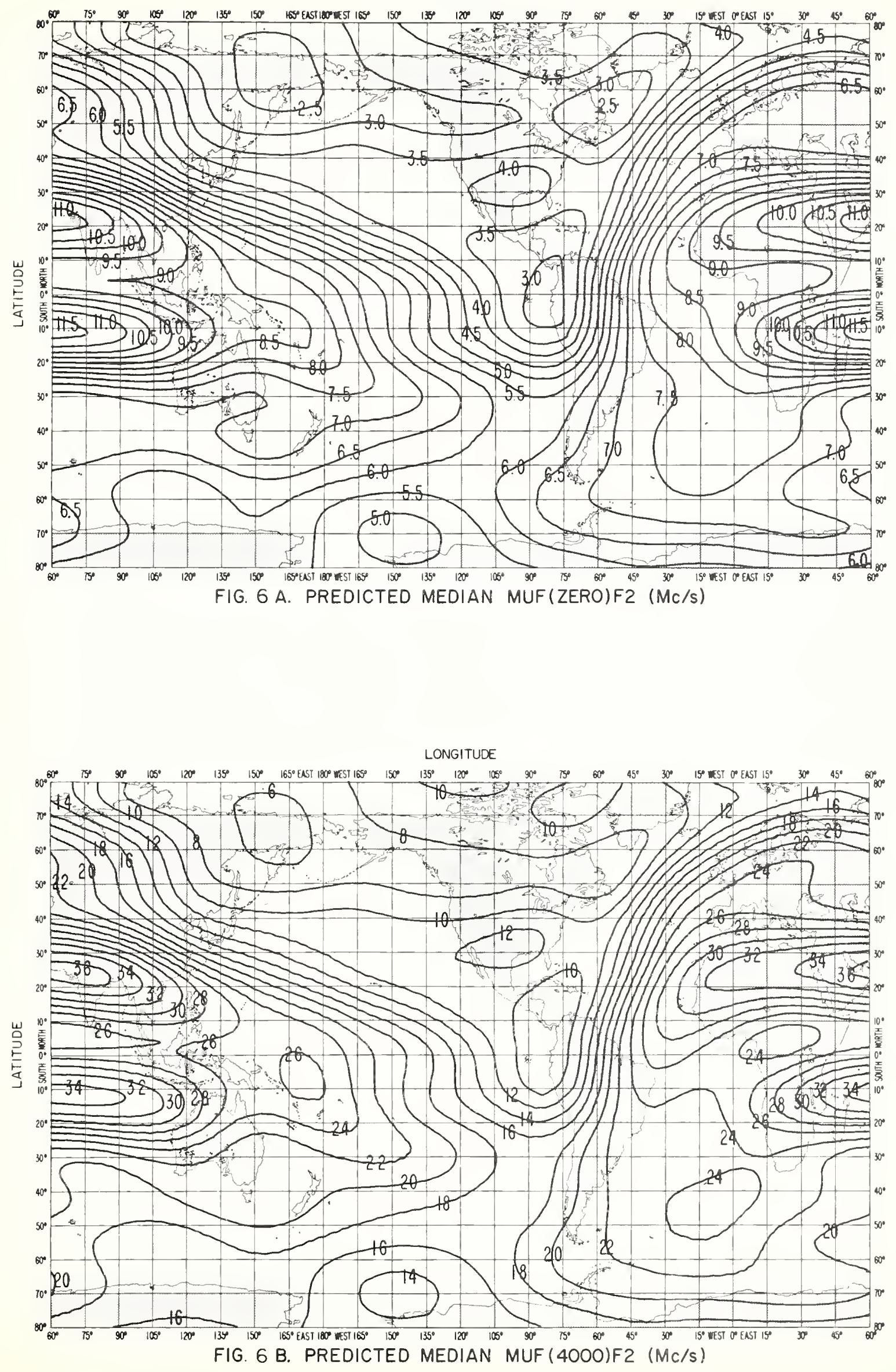


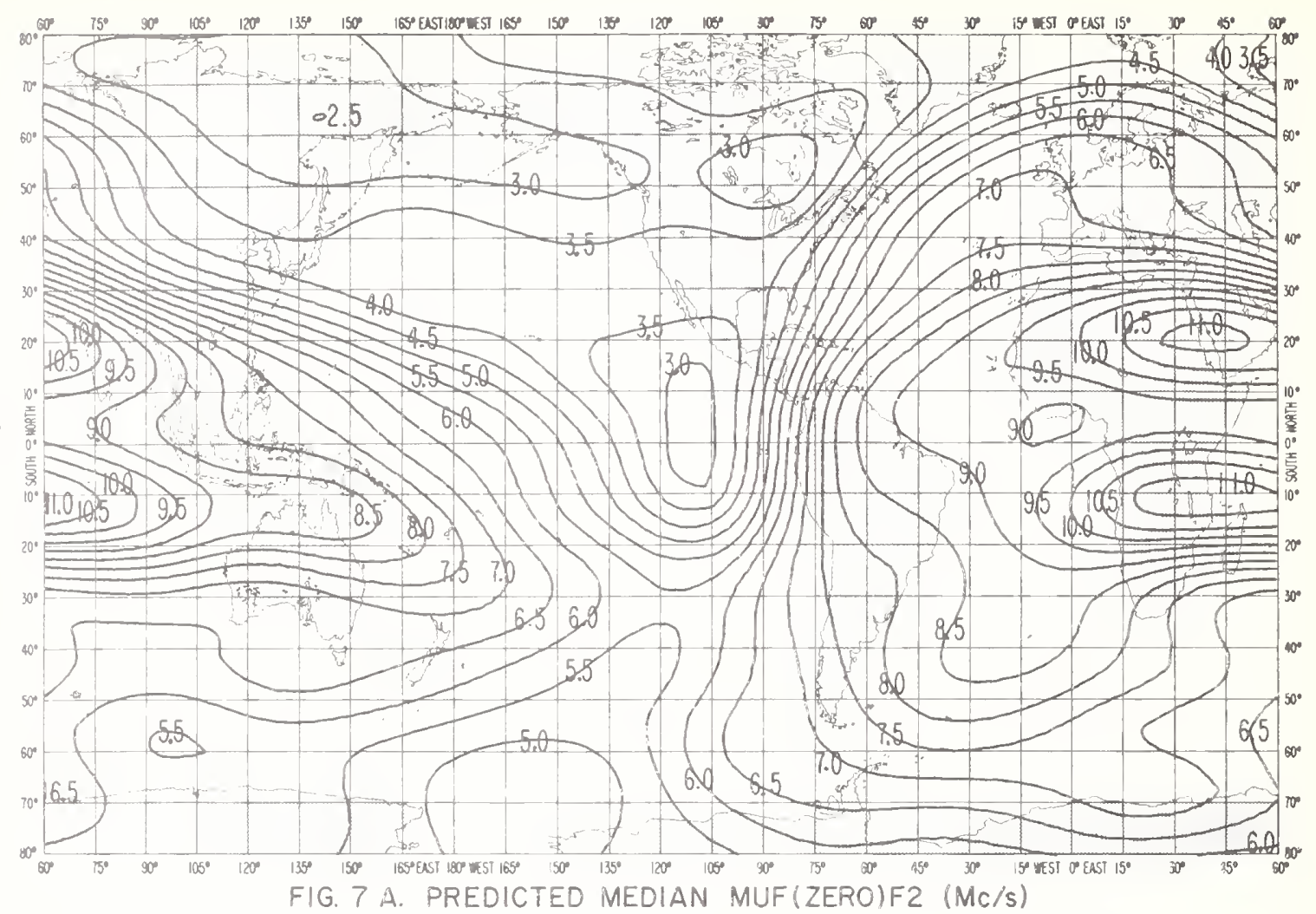

LONGITUDE

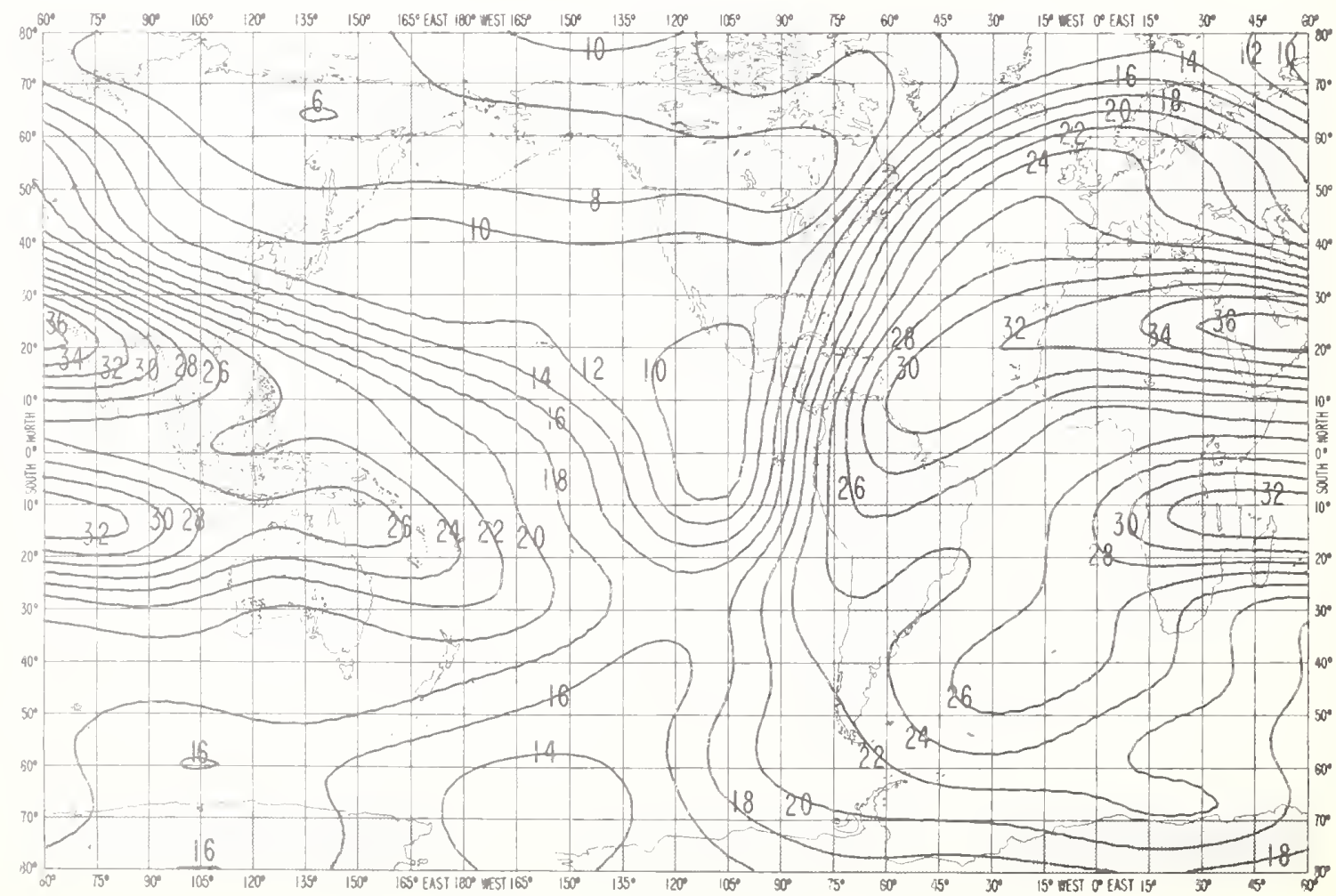

FIG. 7 B. PREDICTED MEDIAN MUF $(4000) \mathrm{F} 2(\mathrm{Mc} / \mathrm{s})$ 


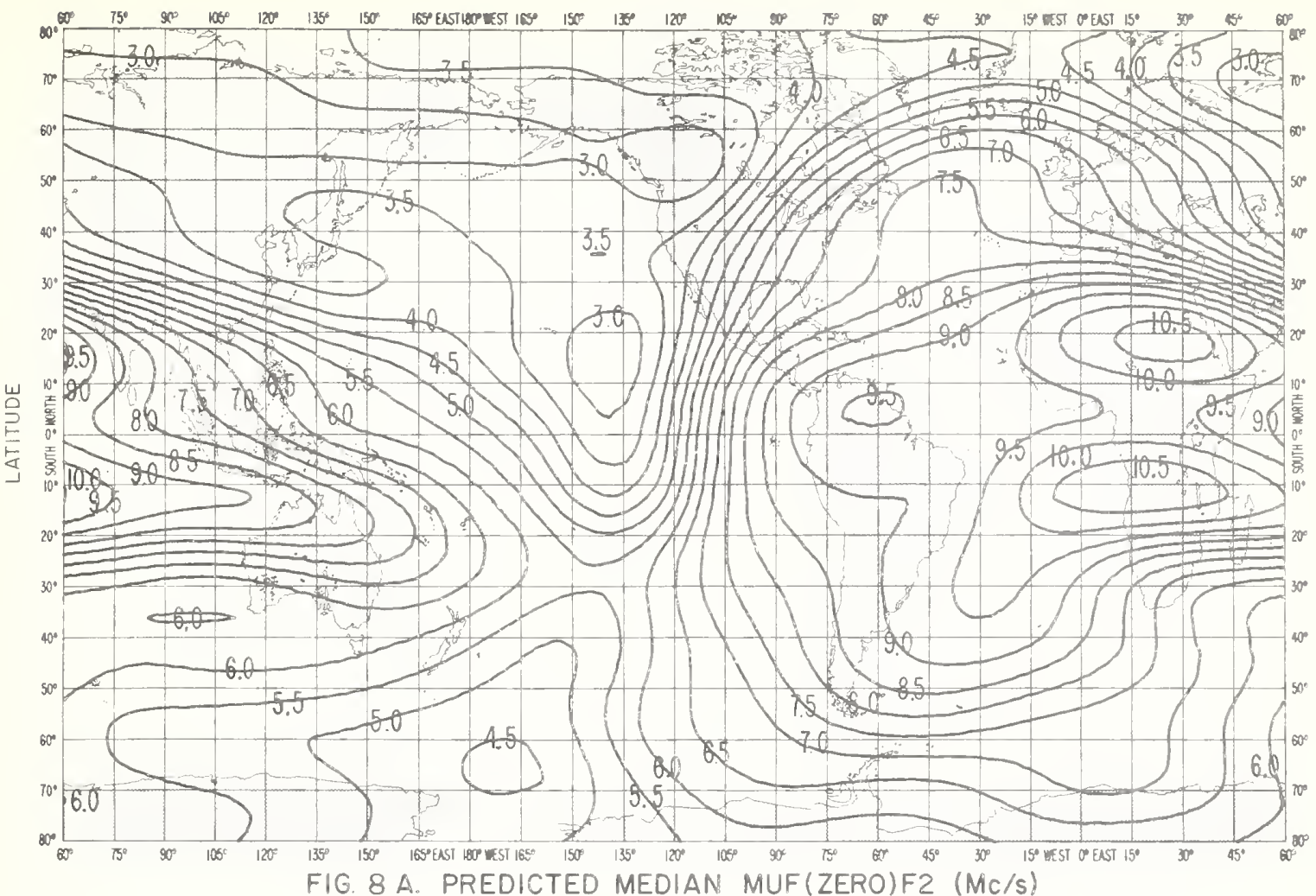

LONGITUDE
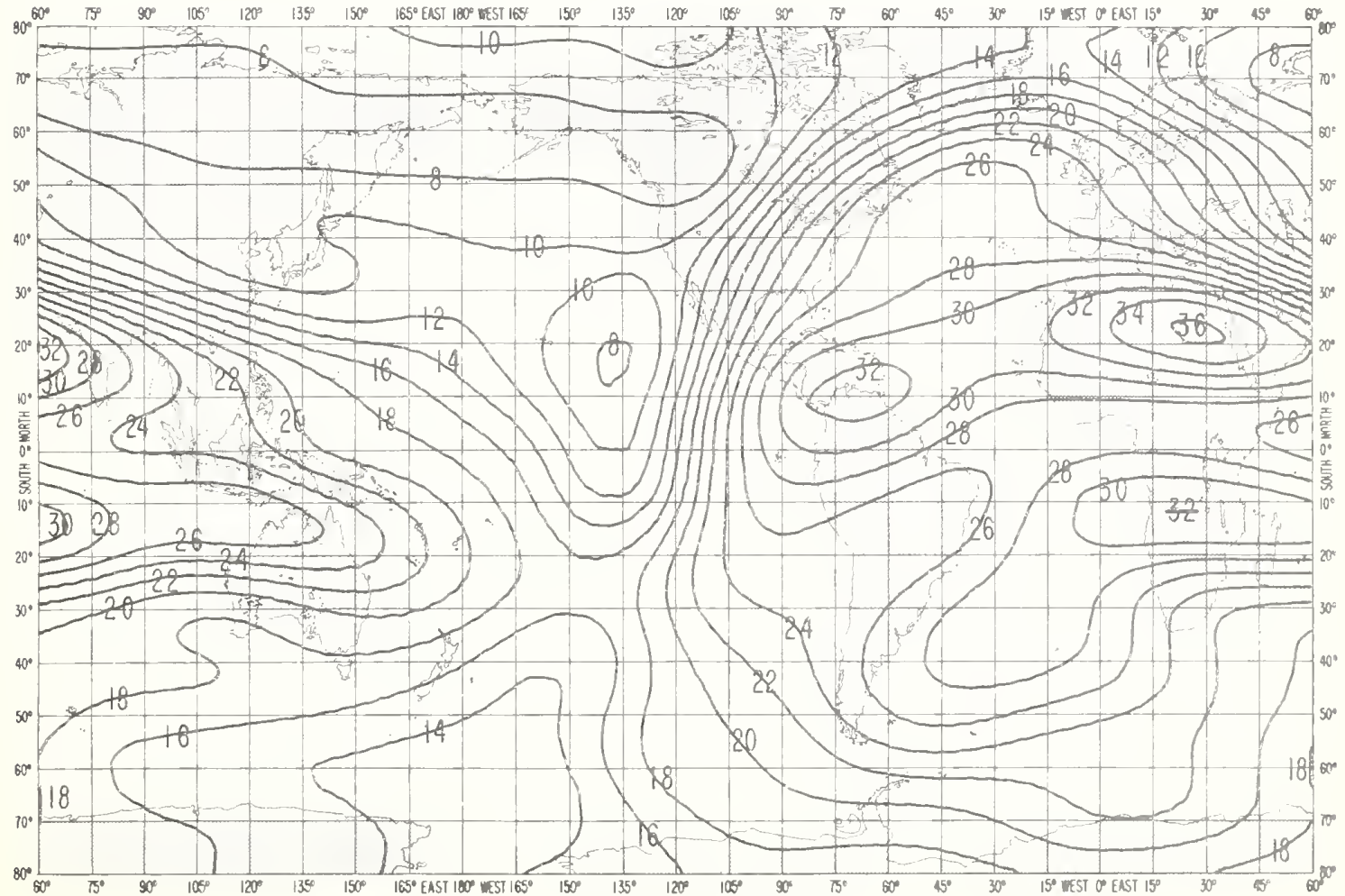

FIG. 8 E. PREDICTED MEDIAN MUF $(4000) \mathrm{F} 2(\mathrm{Mc} / \mathrm{s})$ 


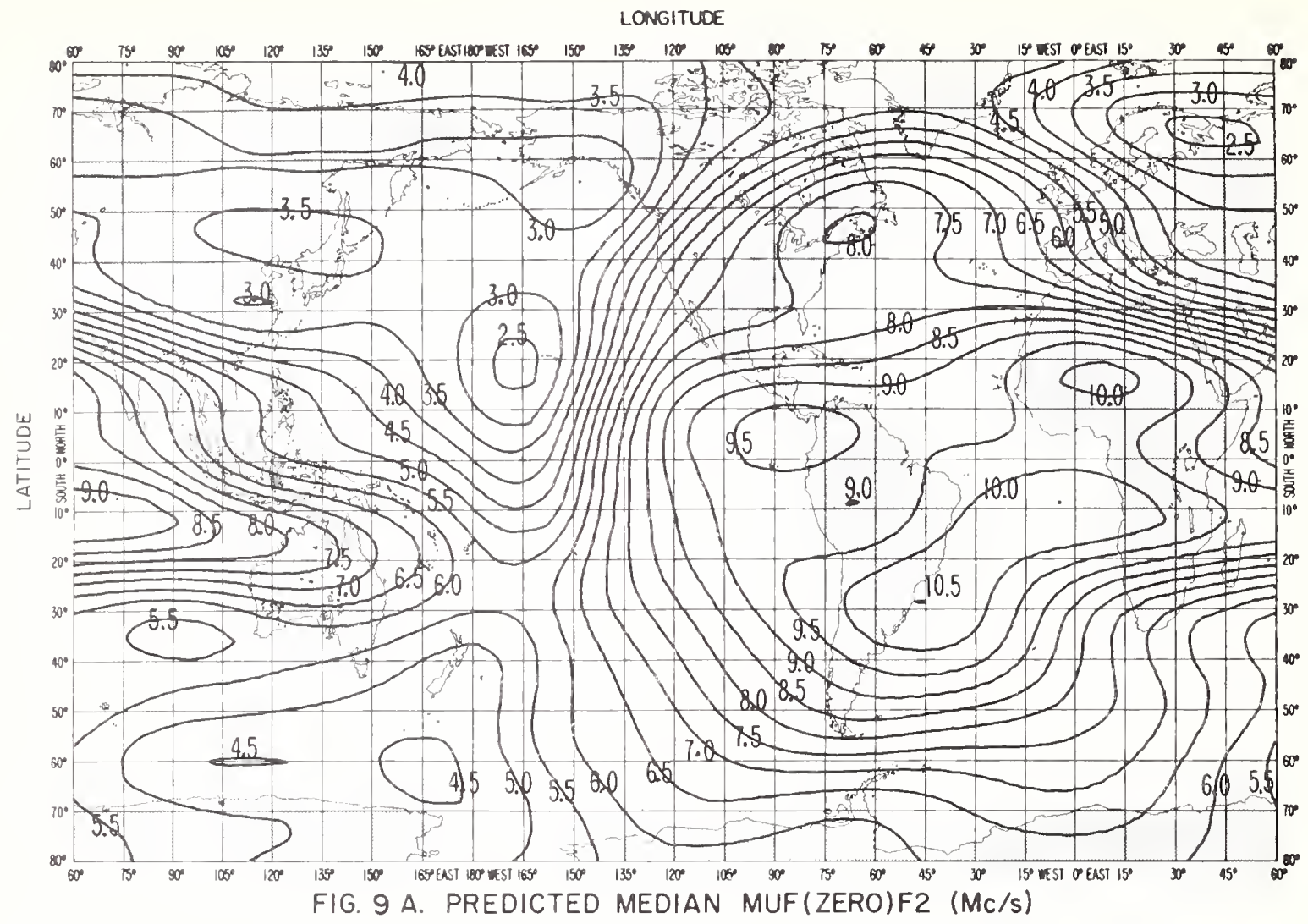

LONGITUDE

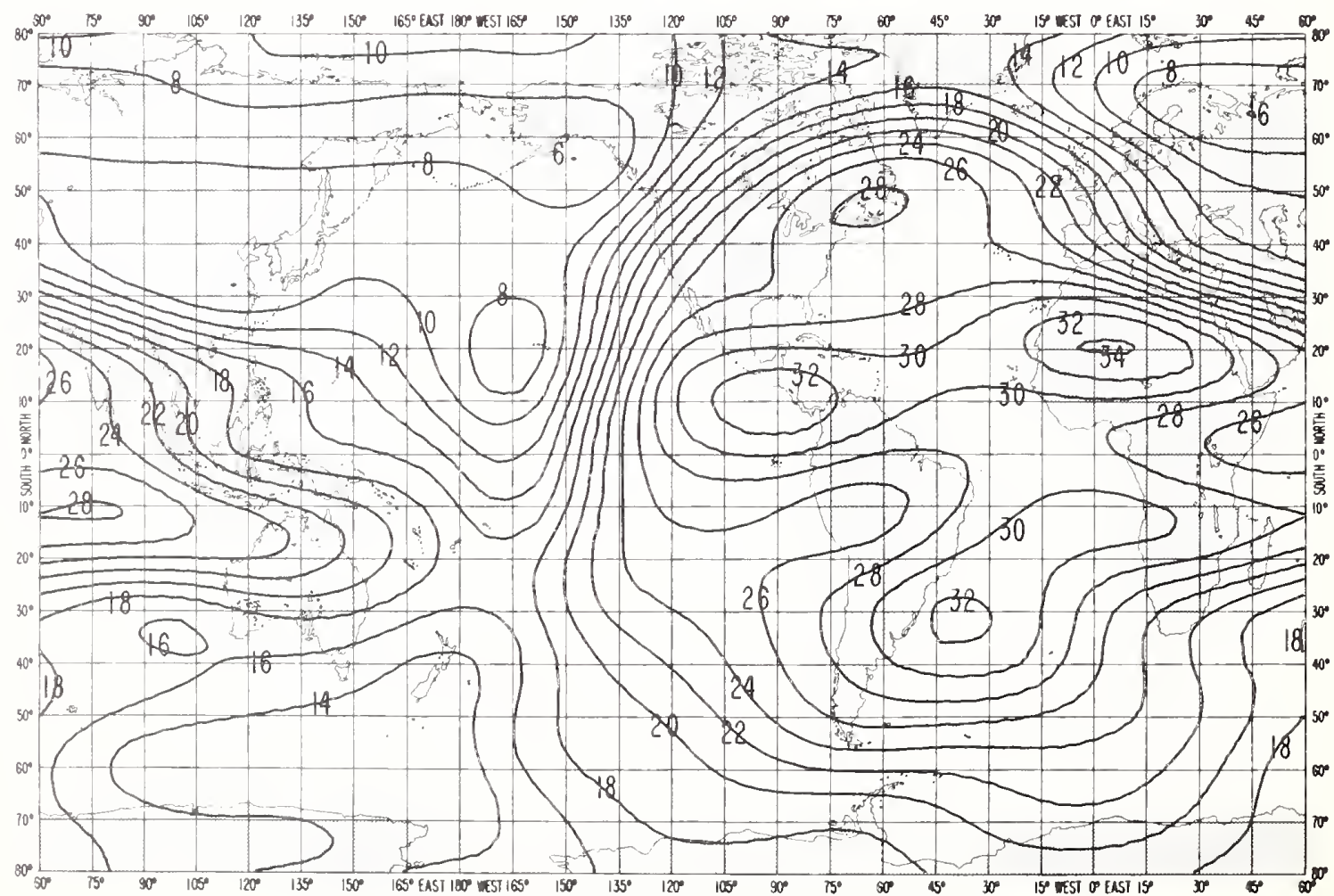

FIG. 9 B. PREDICTED MEDIAN MUF (4000)F2 (Mc/s) 


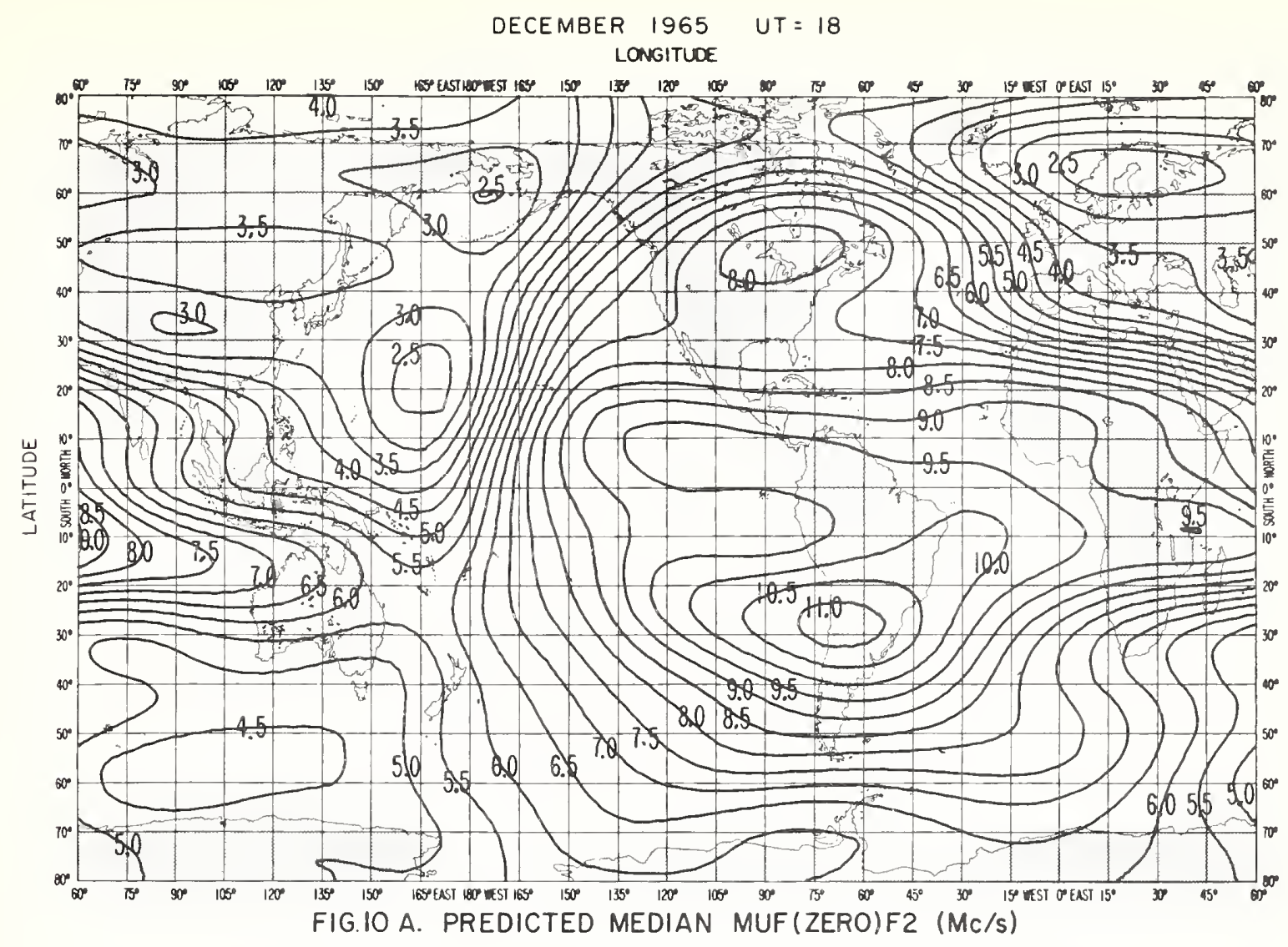

LONGITUDE

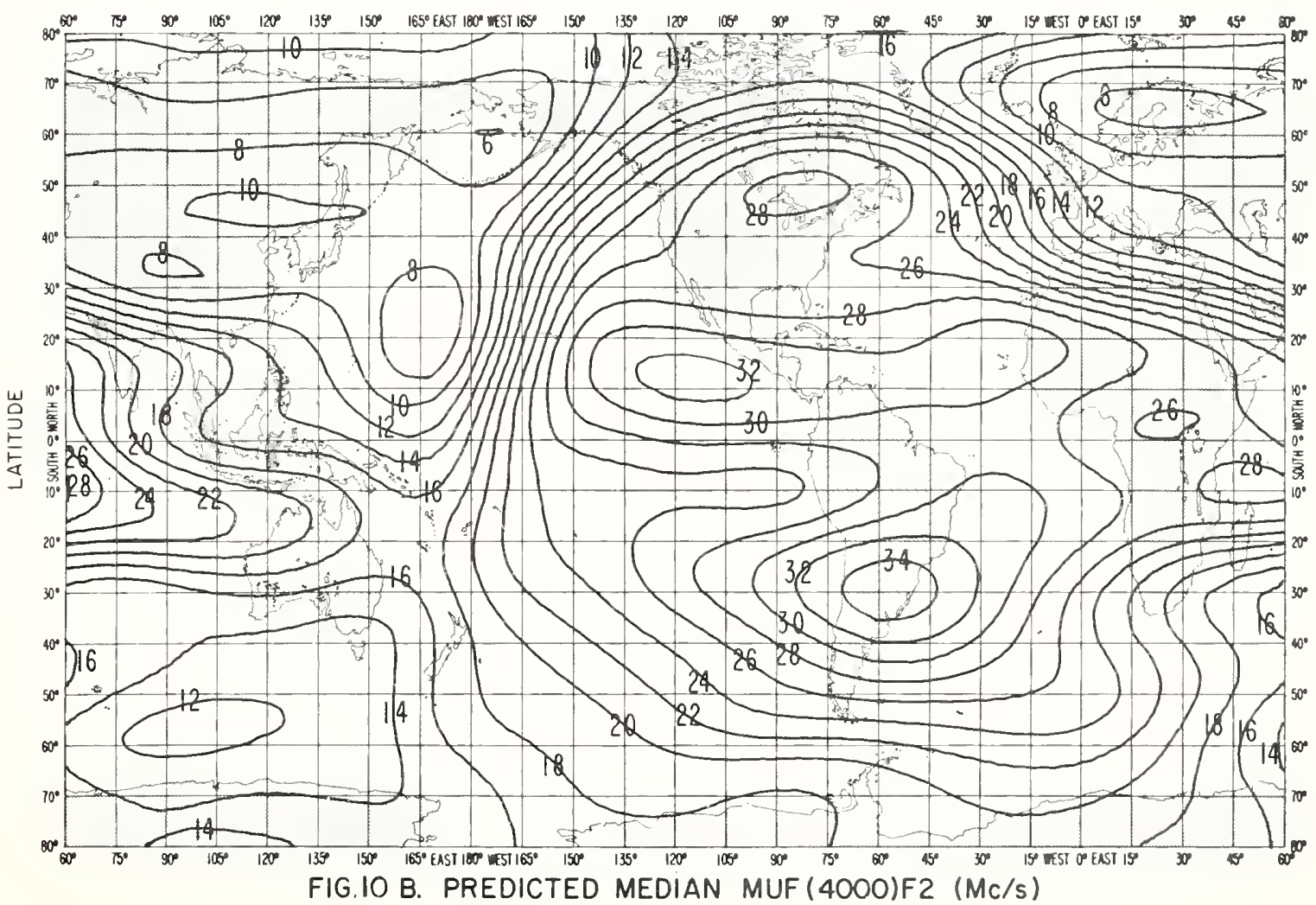




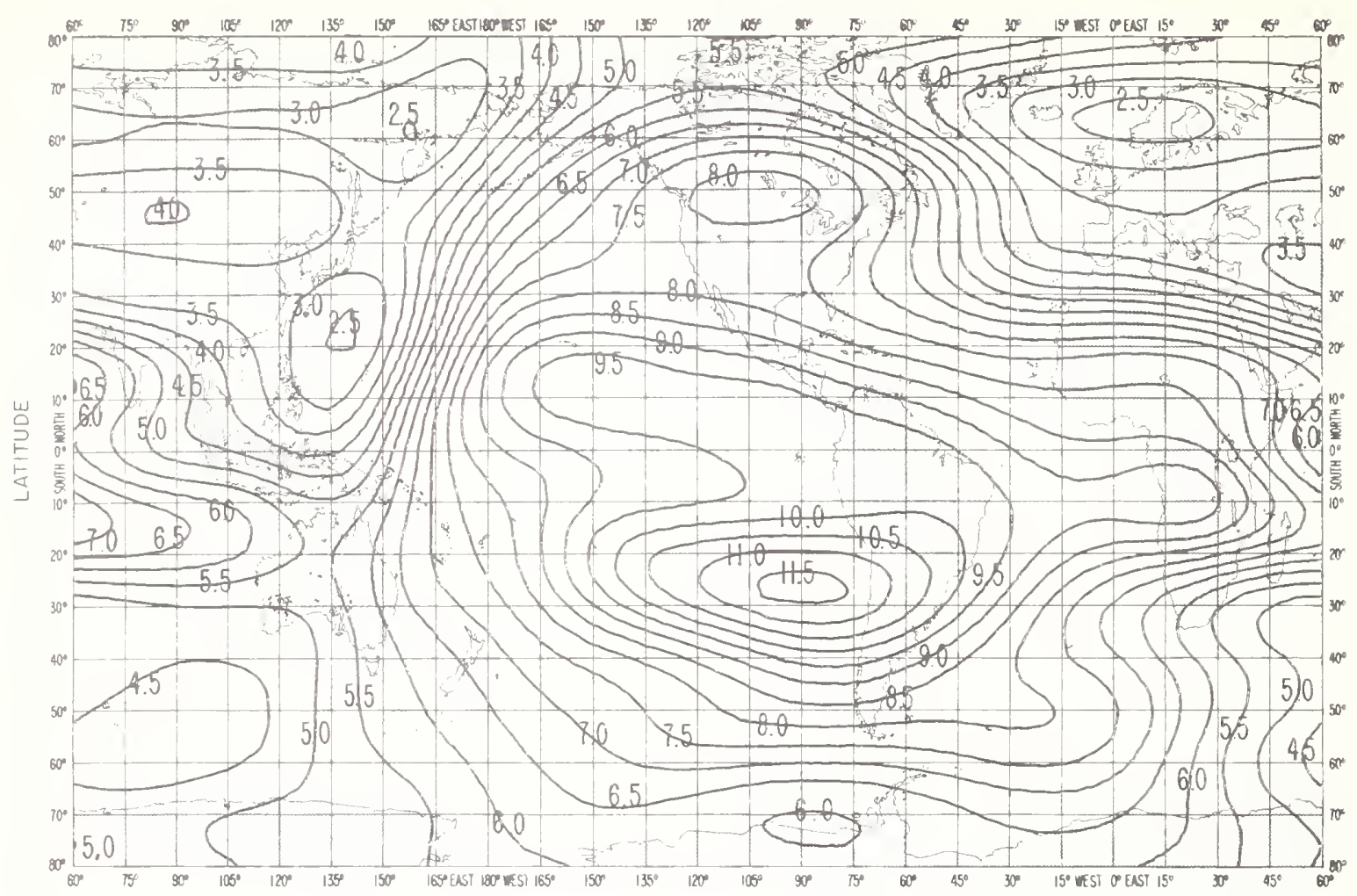

FIG $\|$ A. PREDICTED MEDIAN MUF(ZERO)F2 ( $\mathrm{Mc} / \mathrm{s}$ )

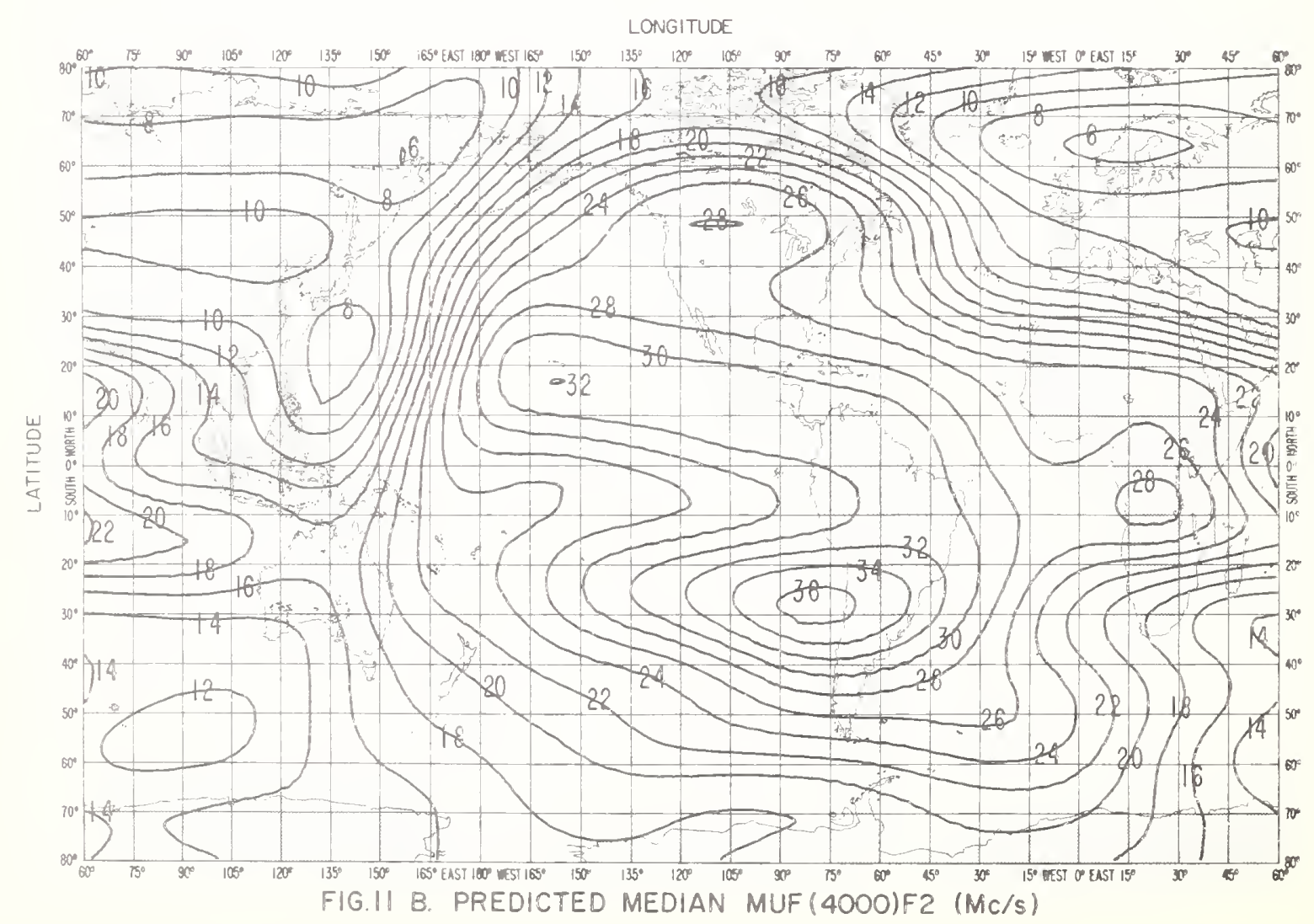




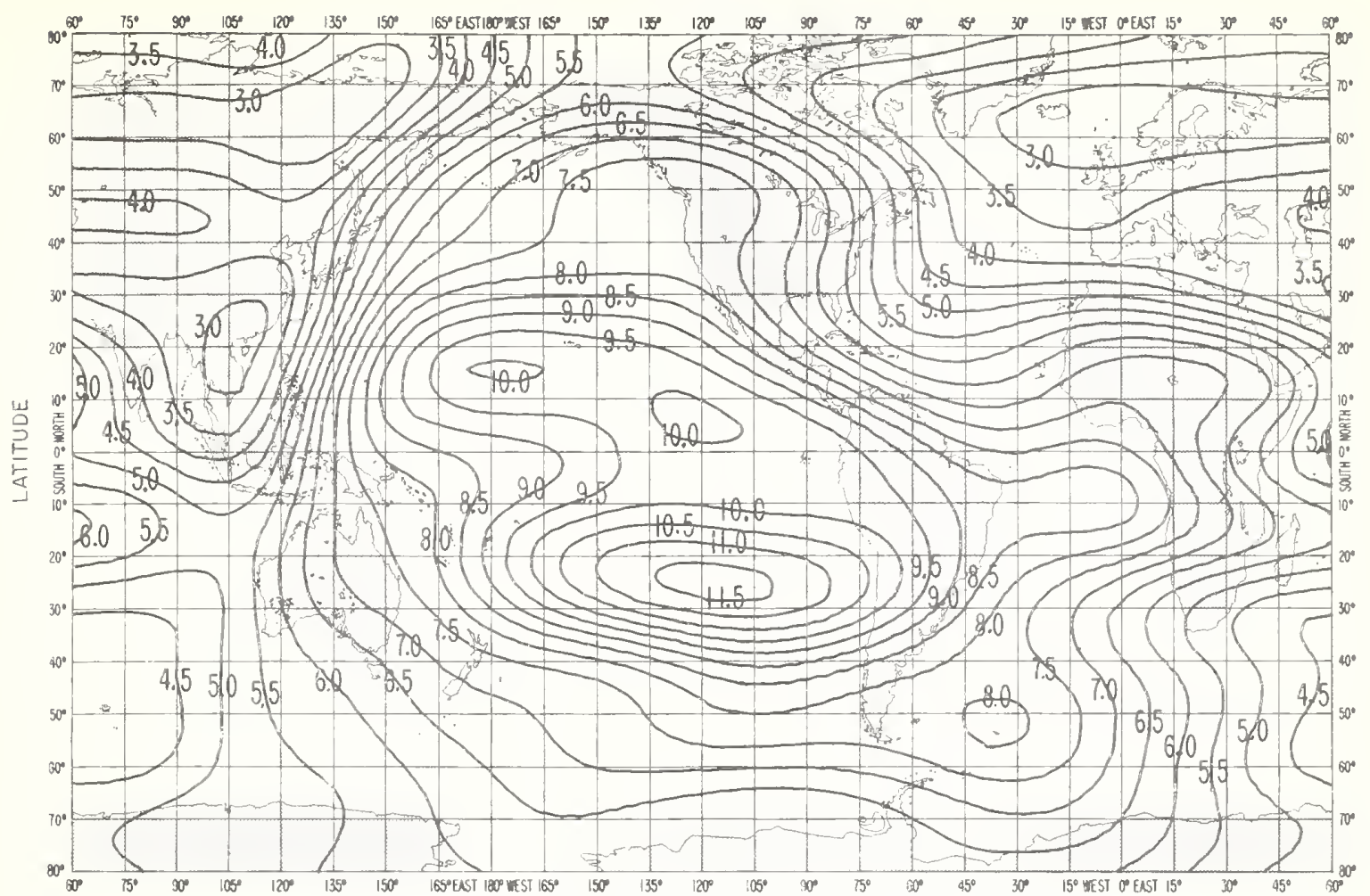

FIG.I2 A. PREDICTED MEDIAN MUF(ZERO)F2 (Mc/s)

LONGITUDE

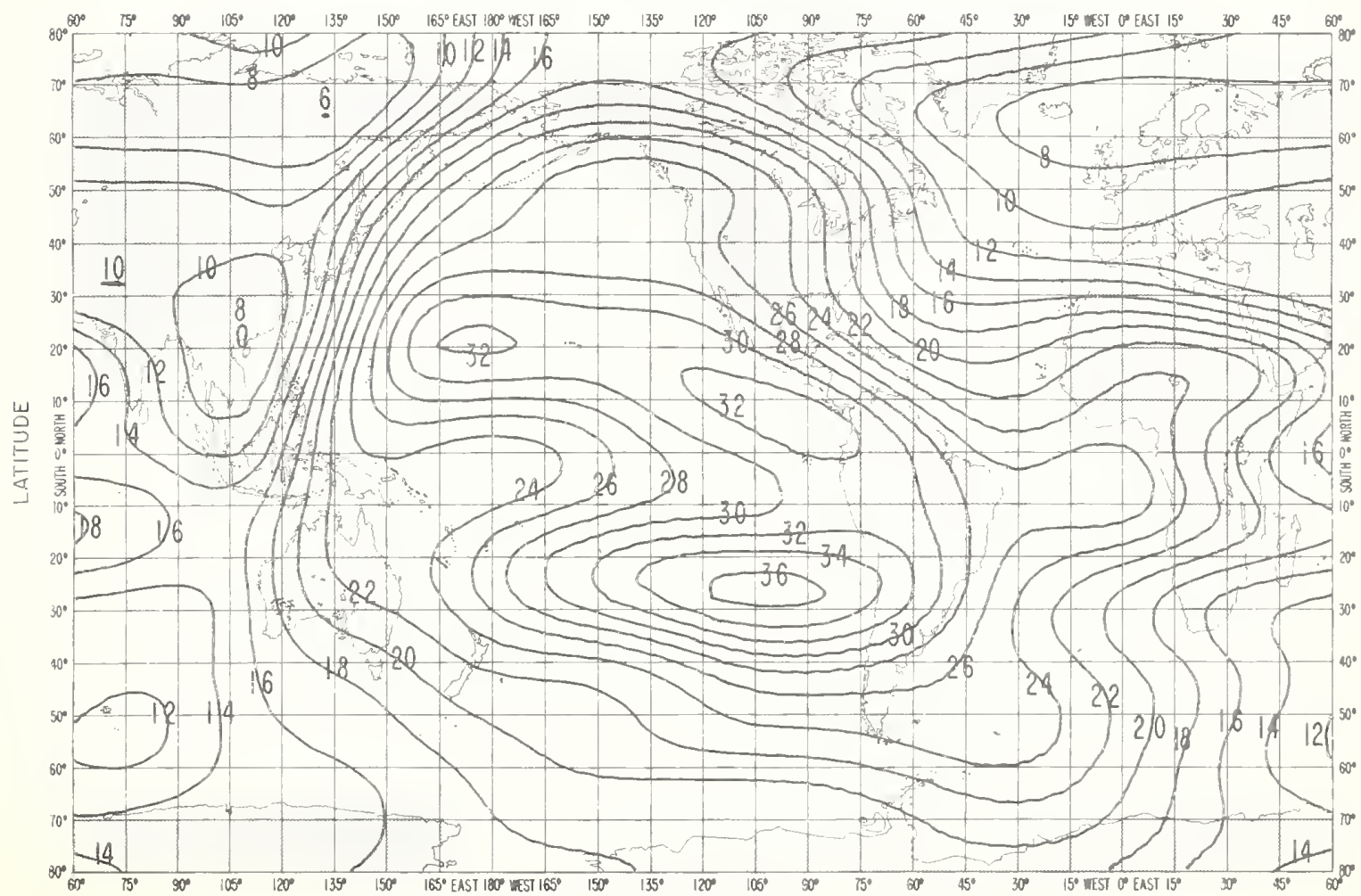

FIG.I2 B. PREDICTED MEDIAN MUF (4000) $)_{2}(\mathrm{Mc} / \mathrm{s})$ 
NORTH POLAR AREA

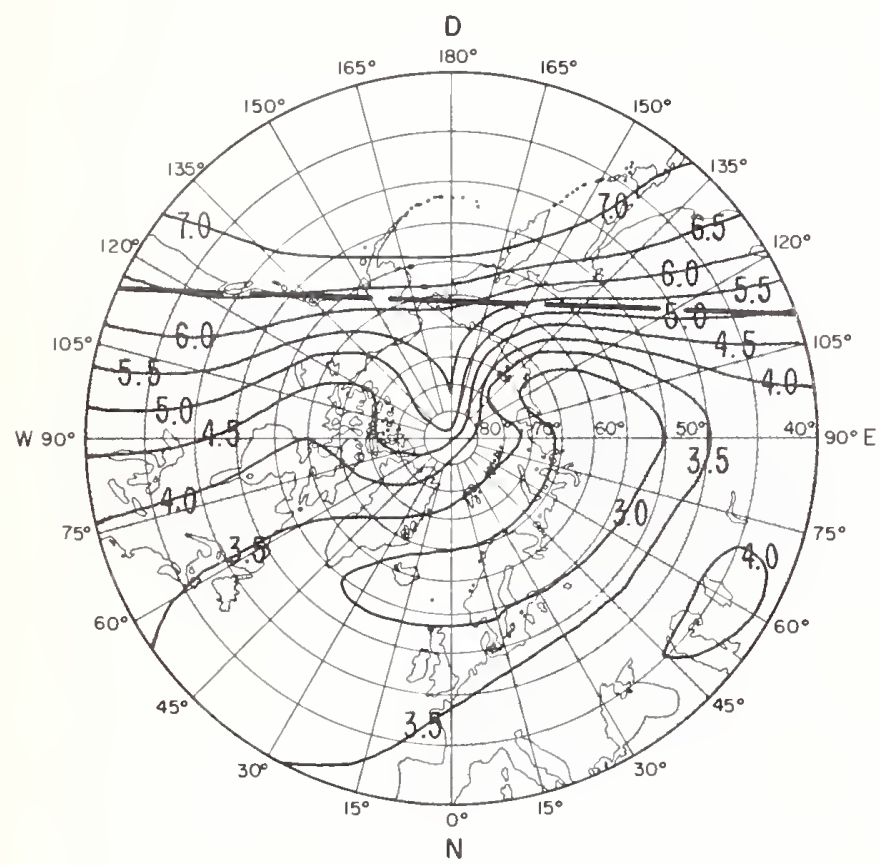

SOUTH POLAR AREA

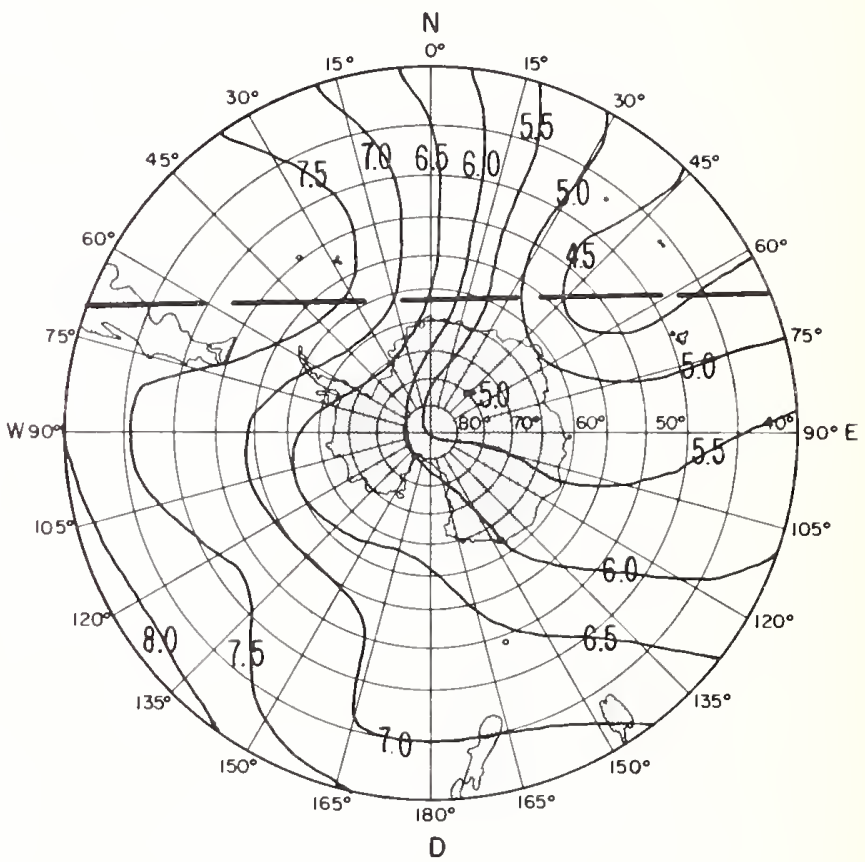

FIG. I 3 A. PREDICTED MEDIAN MUF(ZERO)F2 (MC/S)

NORTH POLAR AREA

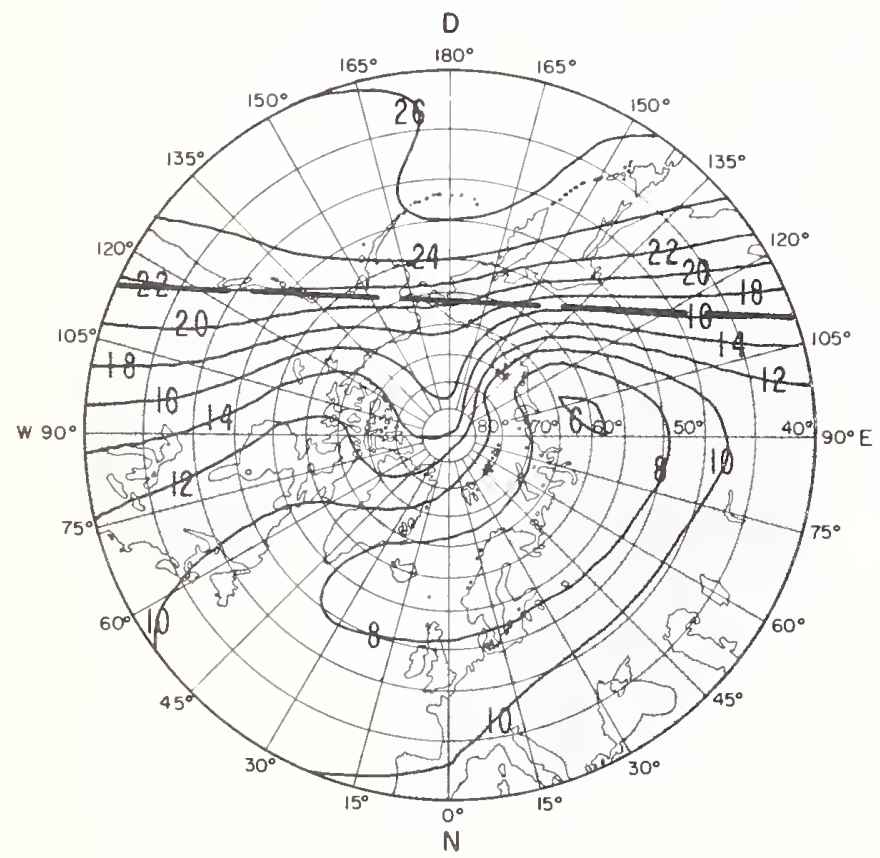

SOUTH POLAR AREA

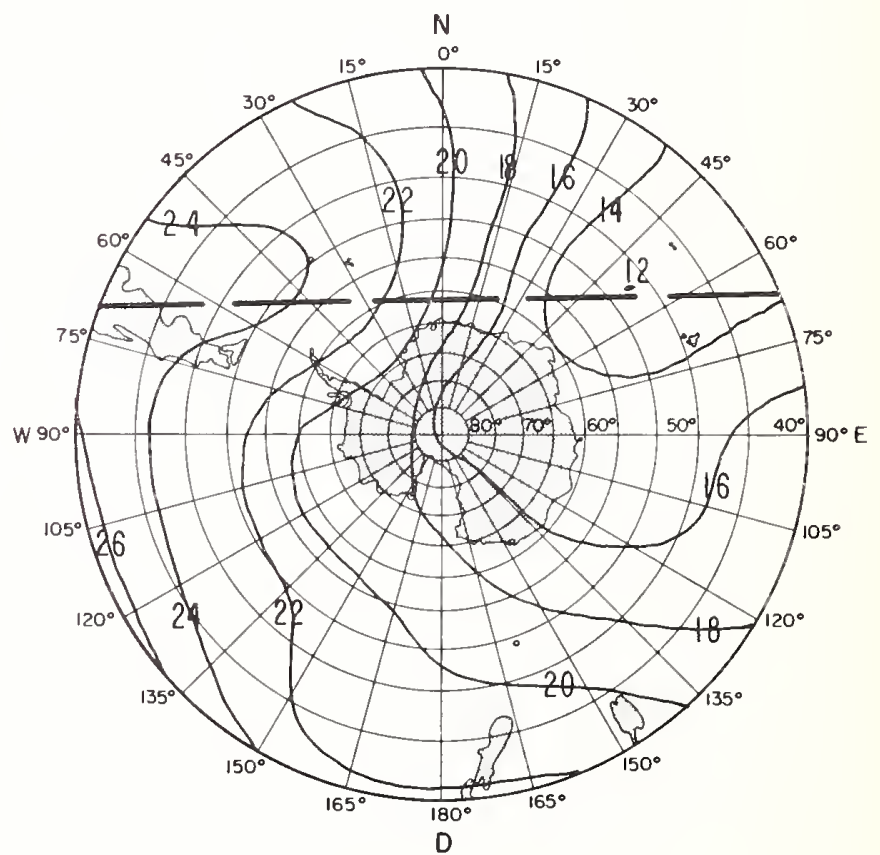



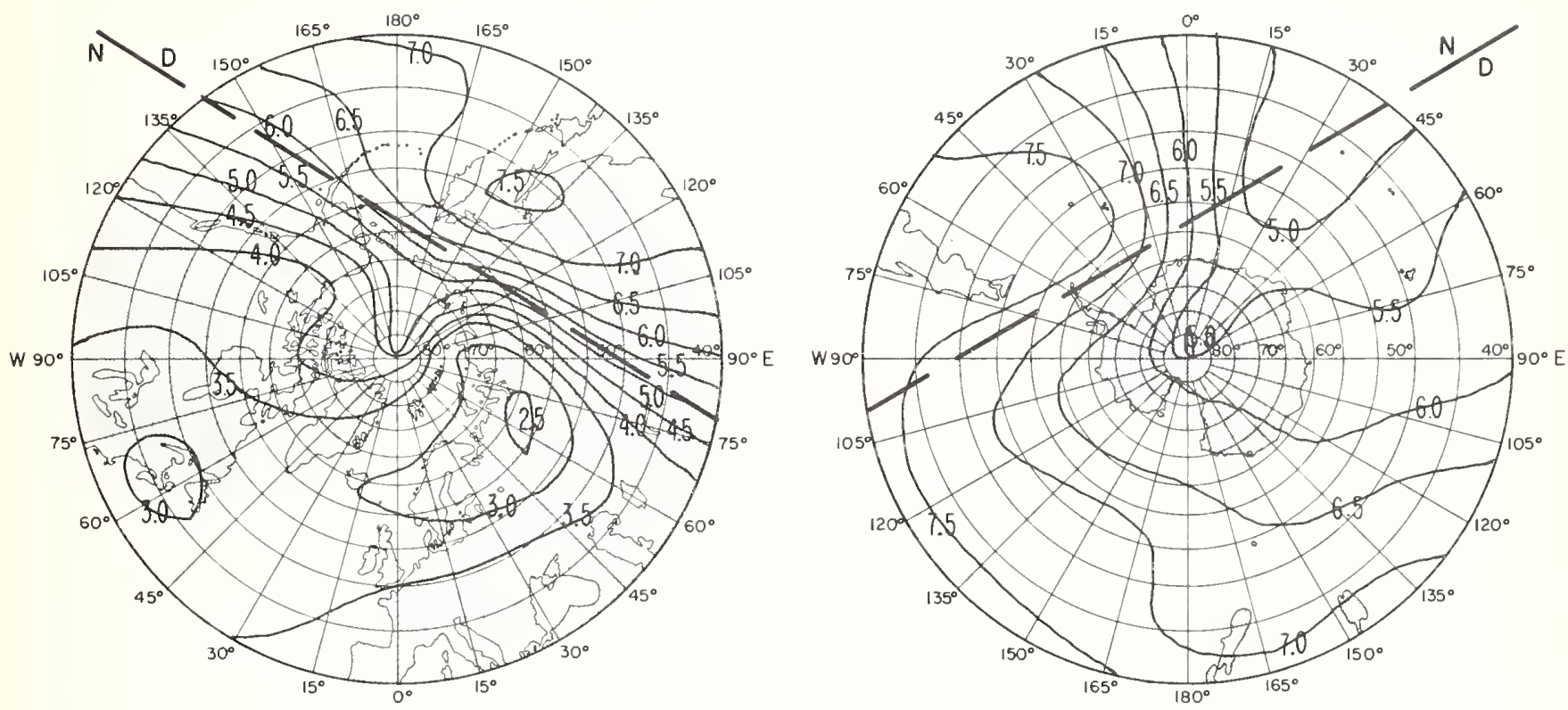

FIG.I4A. PREDICTED MEDIAN MUF(ZERO)F2 (MC/S)
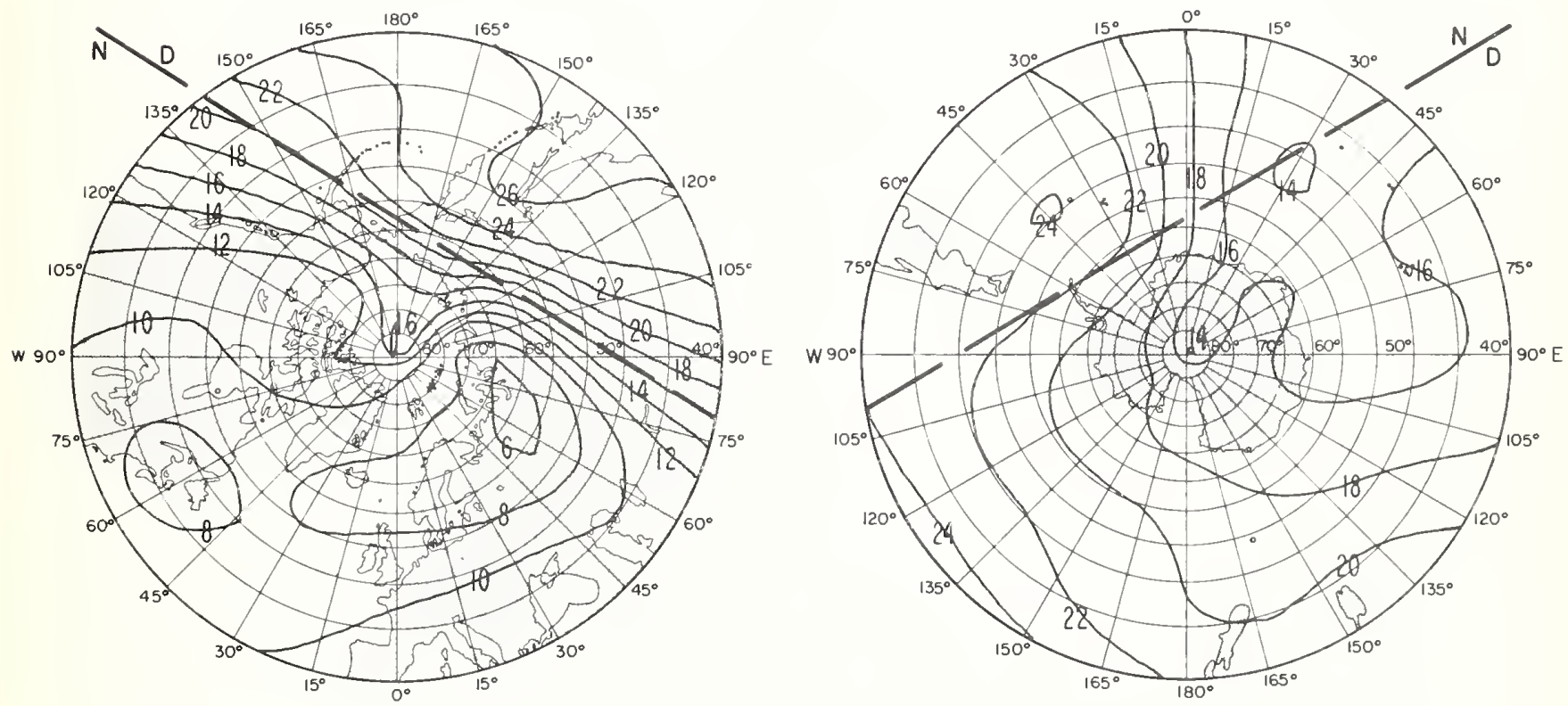

FIG. I 4 B. PREDICTED MEDIAN MUF (4000) F2 (MC/S) 

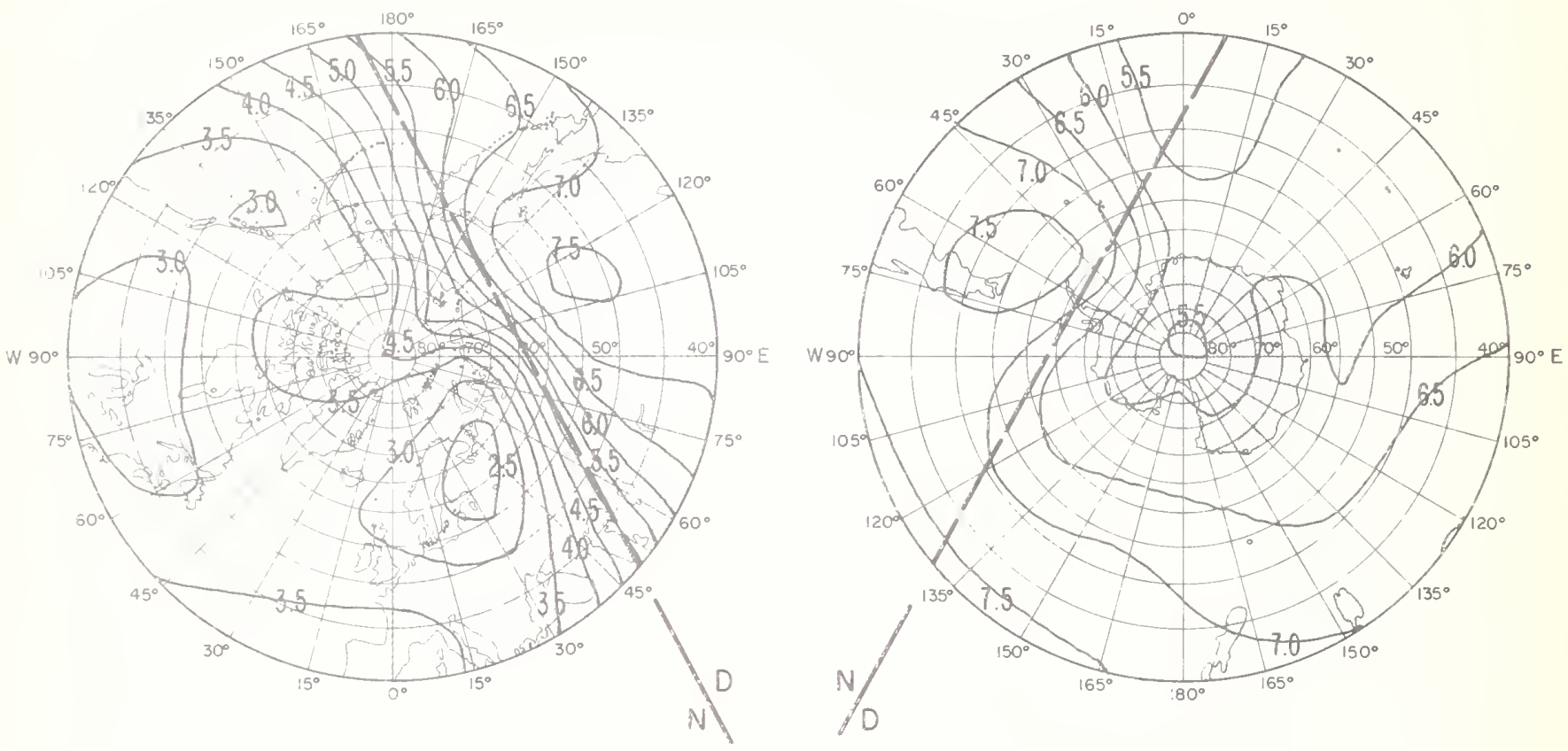

FIG. I5A. PREDICTED MEDIAN MUF(ZERO)F2 (MC/S)
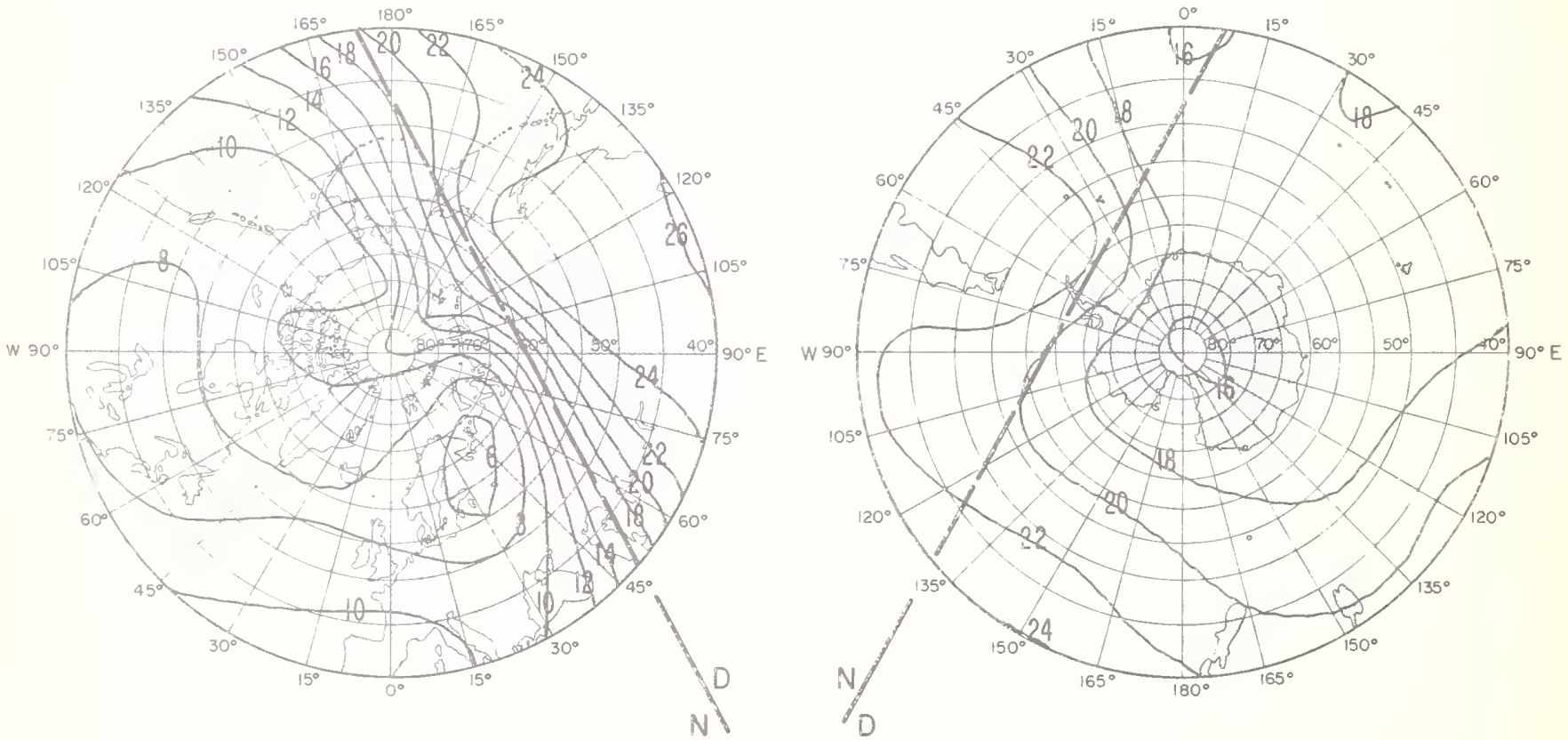

FIG. I 5B. PREDICTED MEDIAN MUF (4000) F2 (MC/S) 

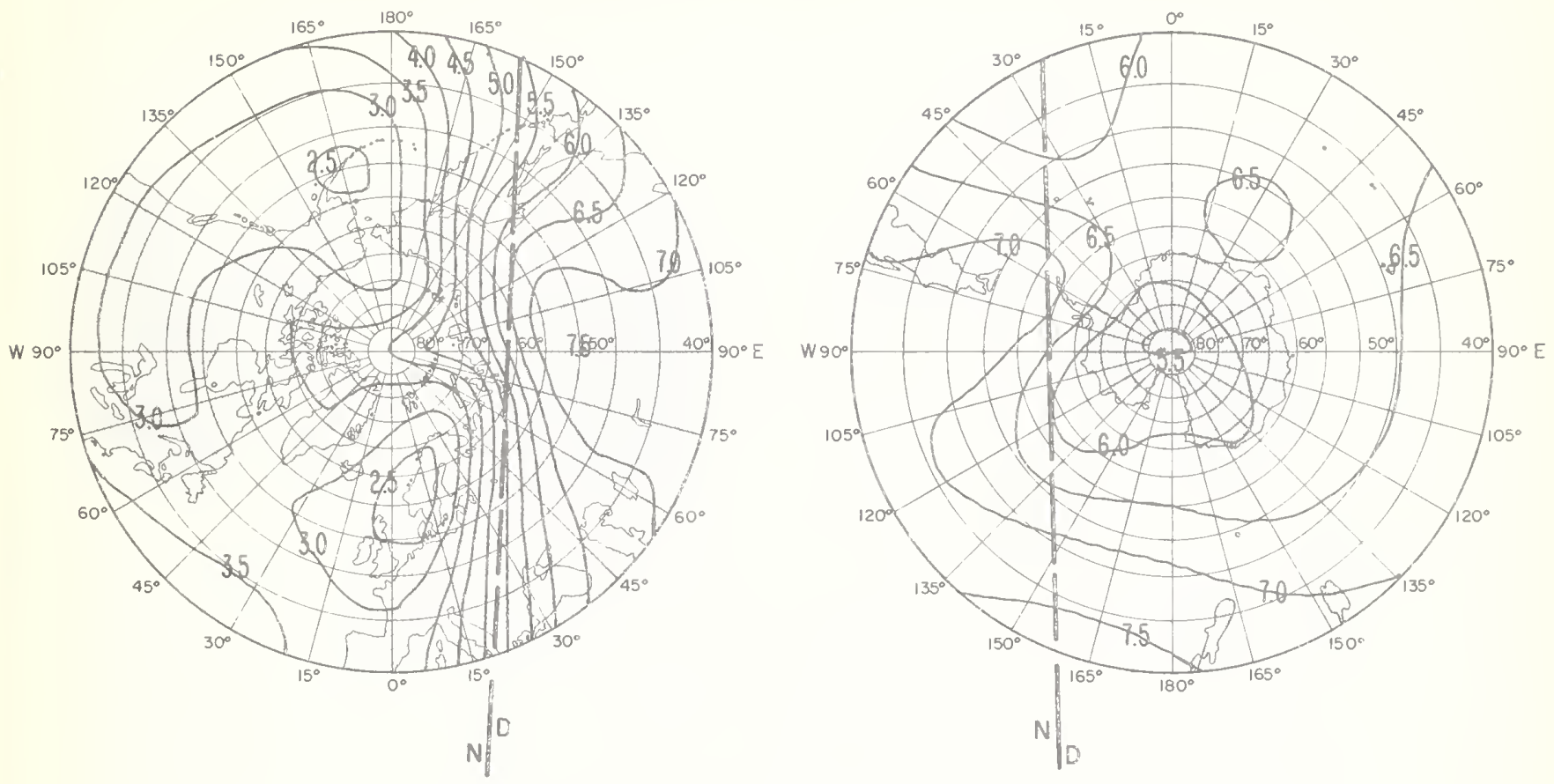

FIG. I6 A. PREDICTED MEDIAN MUF(ZERO)F2 (Mc/S)
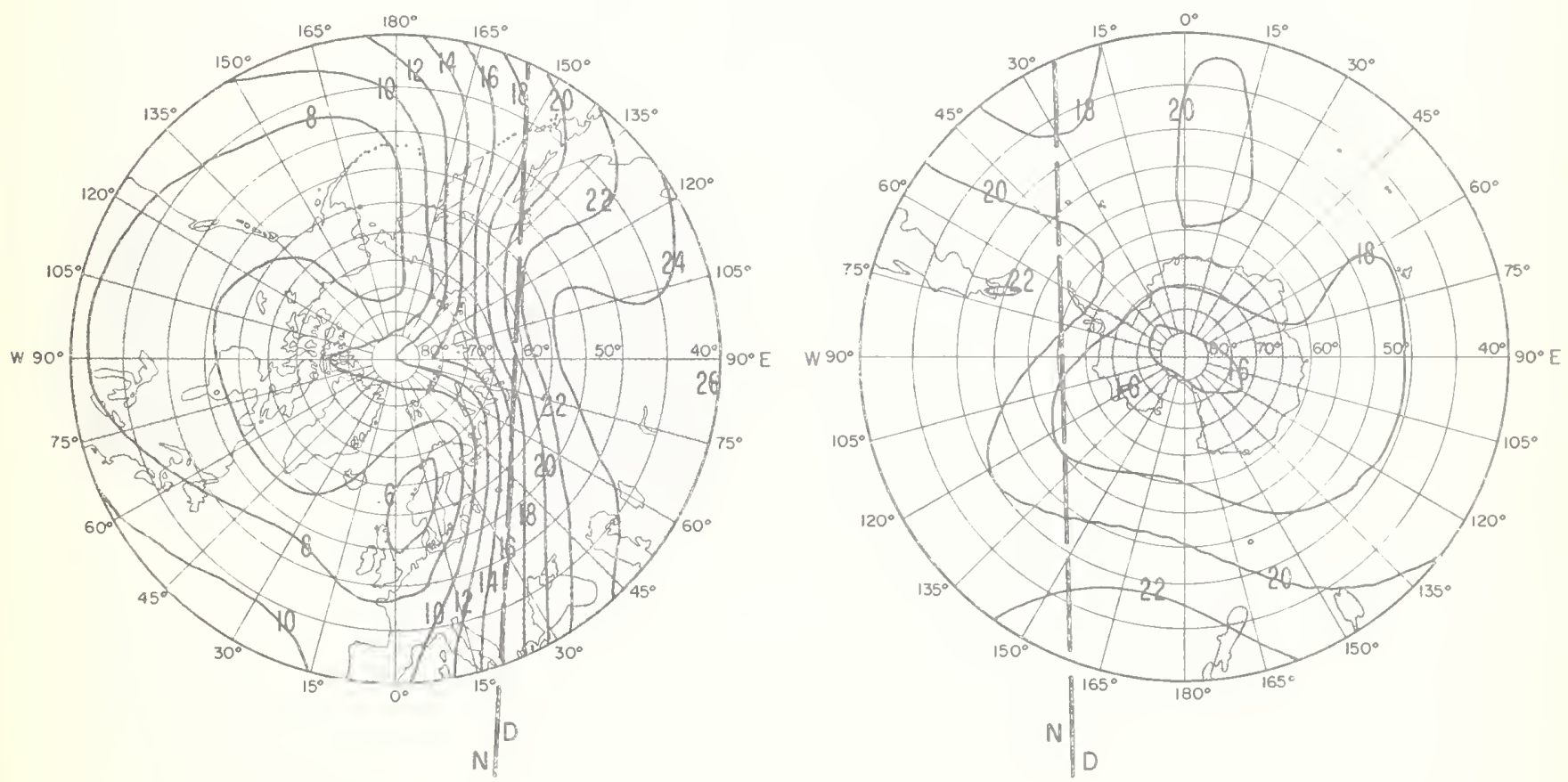

FIG. 16 B. PREDICTED MEDIAN MUF (4000) F2. (MC/S) 

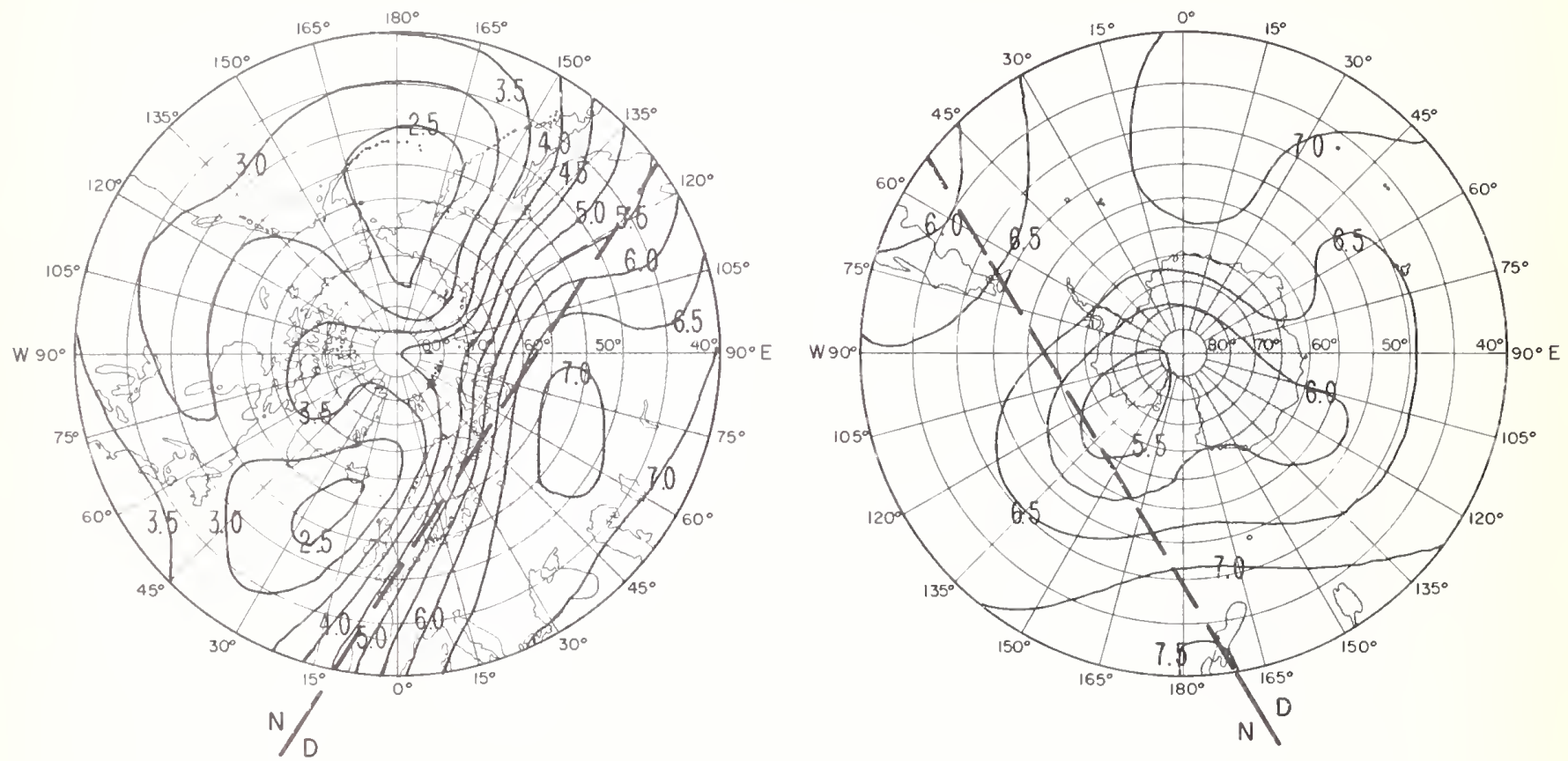

FIG. I7A. PREDICTED MEDIAN MUF(ZERO)F2 (MC/S)

NORTH POLAR AREA

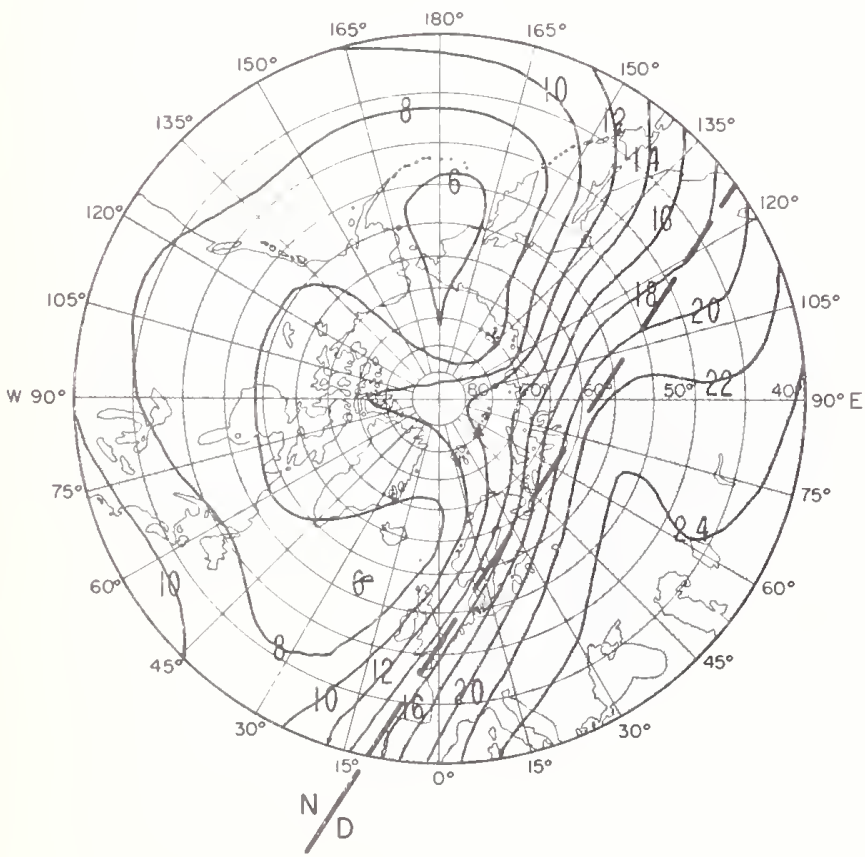

SOUTH POLAR AREA

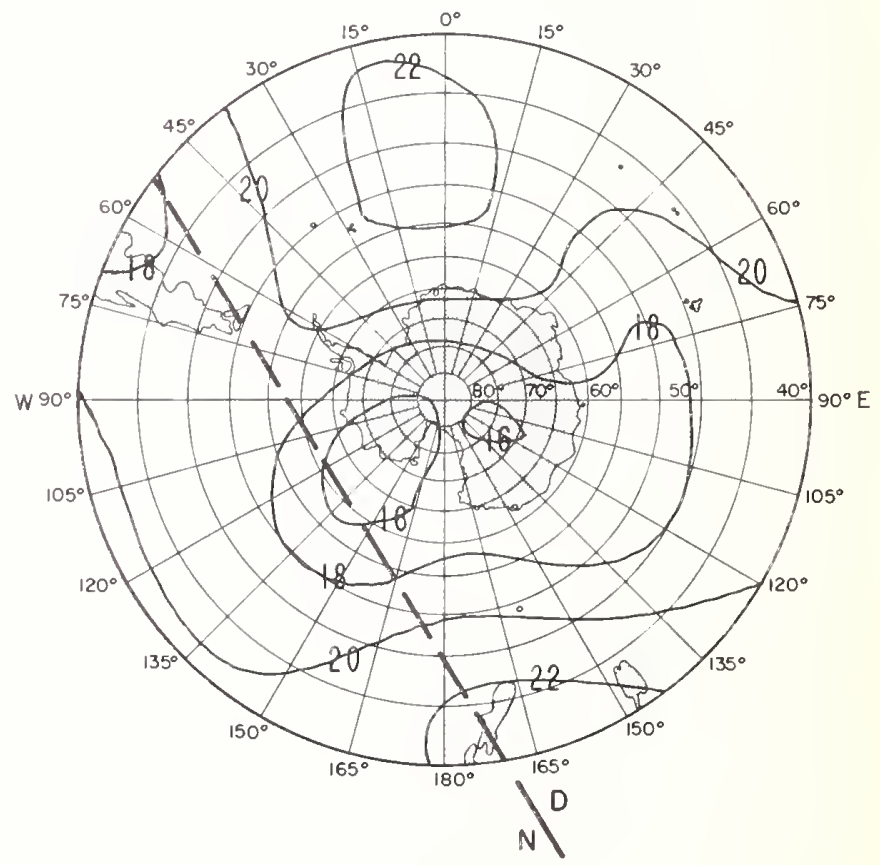



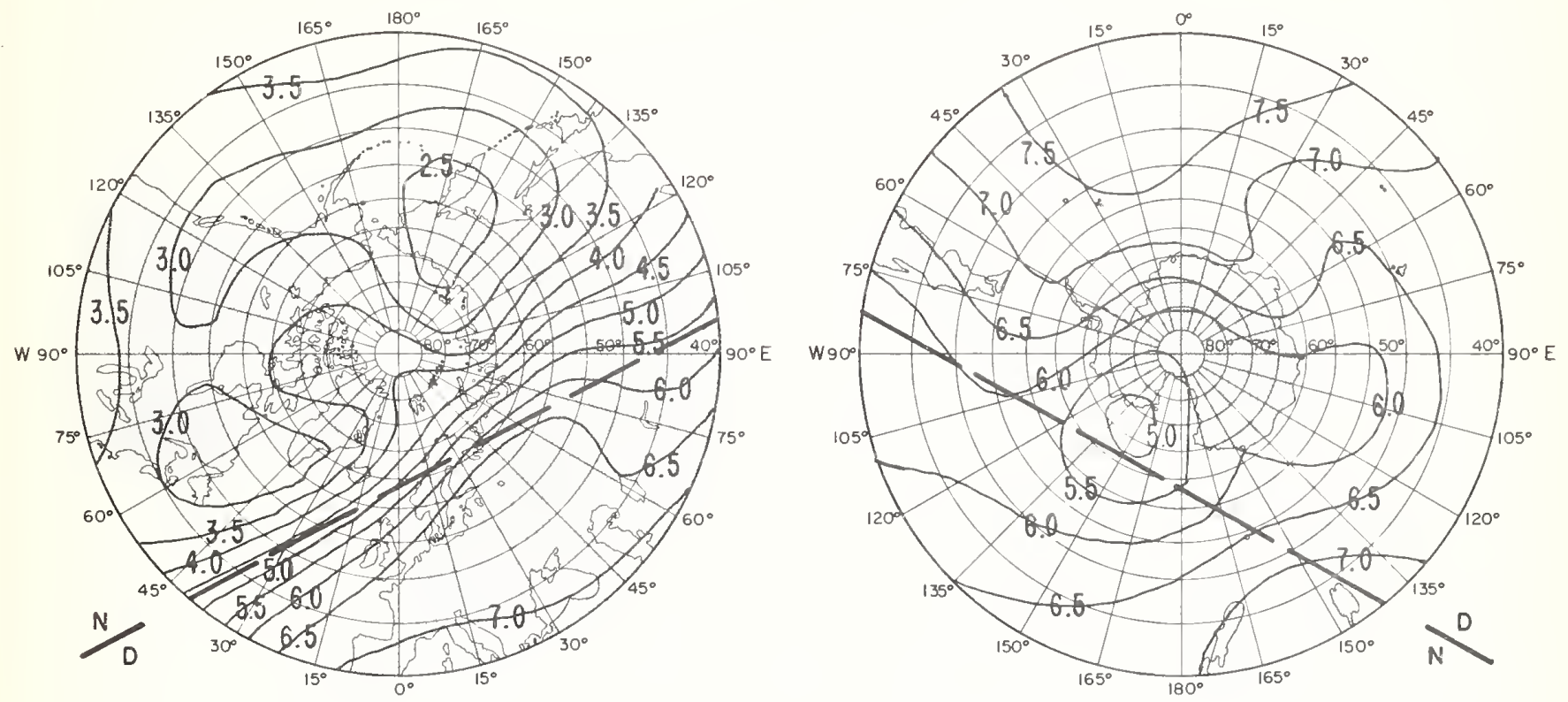

FIG. I8A. PREDICTED MEDIAN MUF(ZERO)F2 (MC/S)

NORTH POLAR AREA

SOUTH POLAR AREA
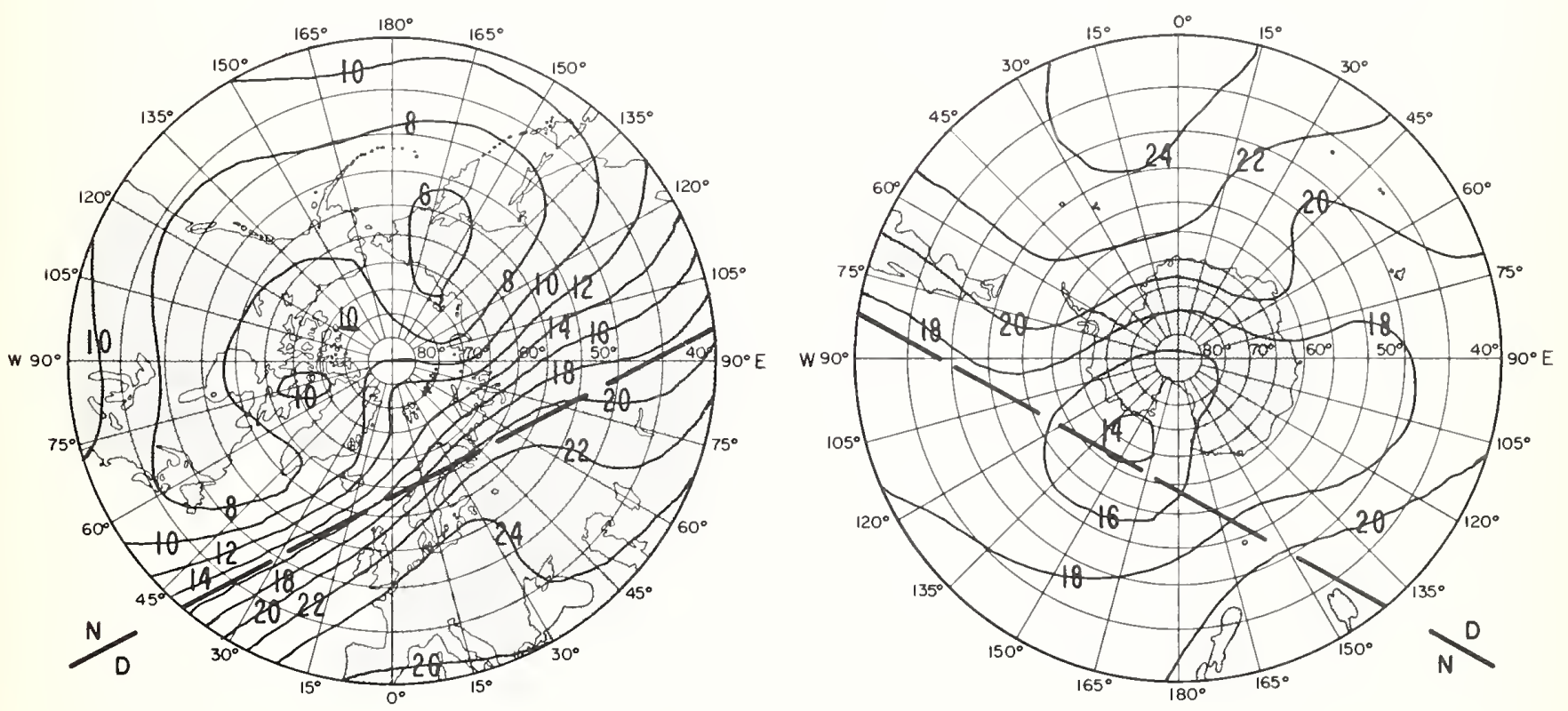
NORTH POLAR AREA

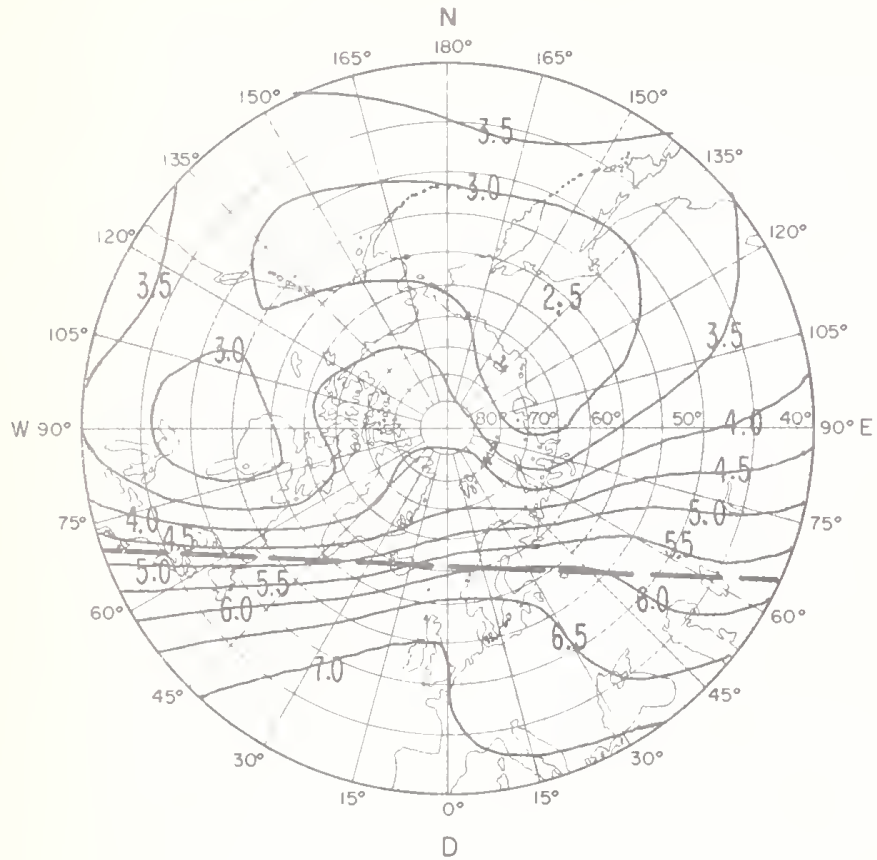

SOUTH POLAR AREA

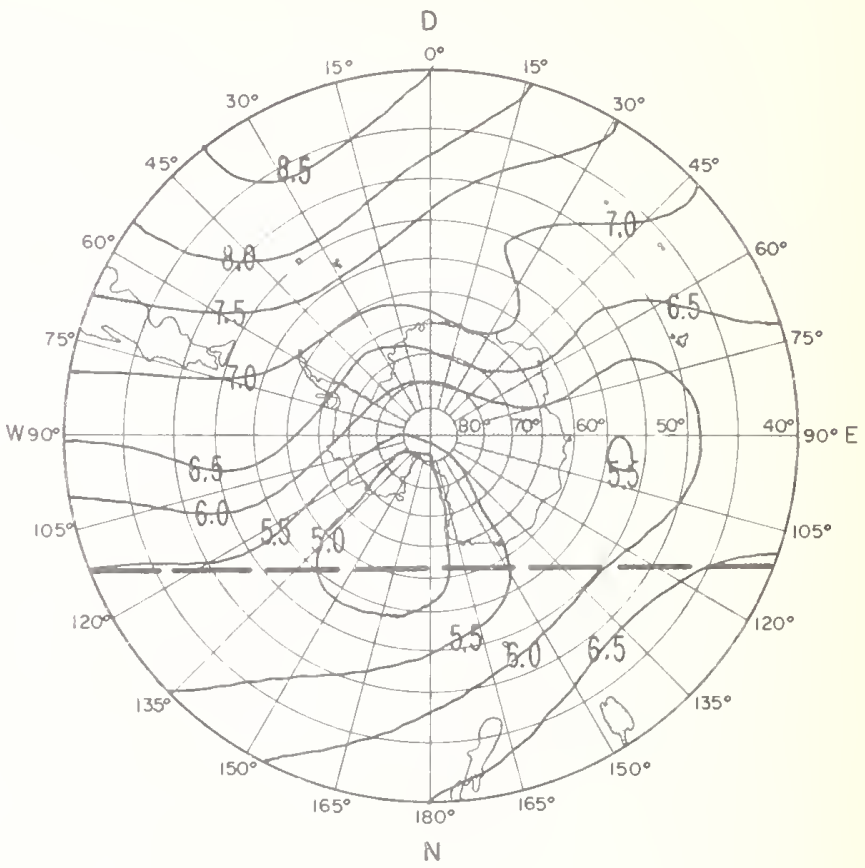

FIG. 19A. PREDICTED MEDIAN MUF(ZERO)F2 (MC/S)

NORTH POLAR AREA

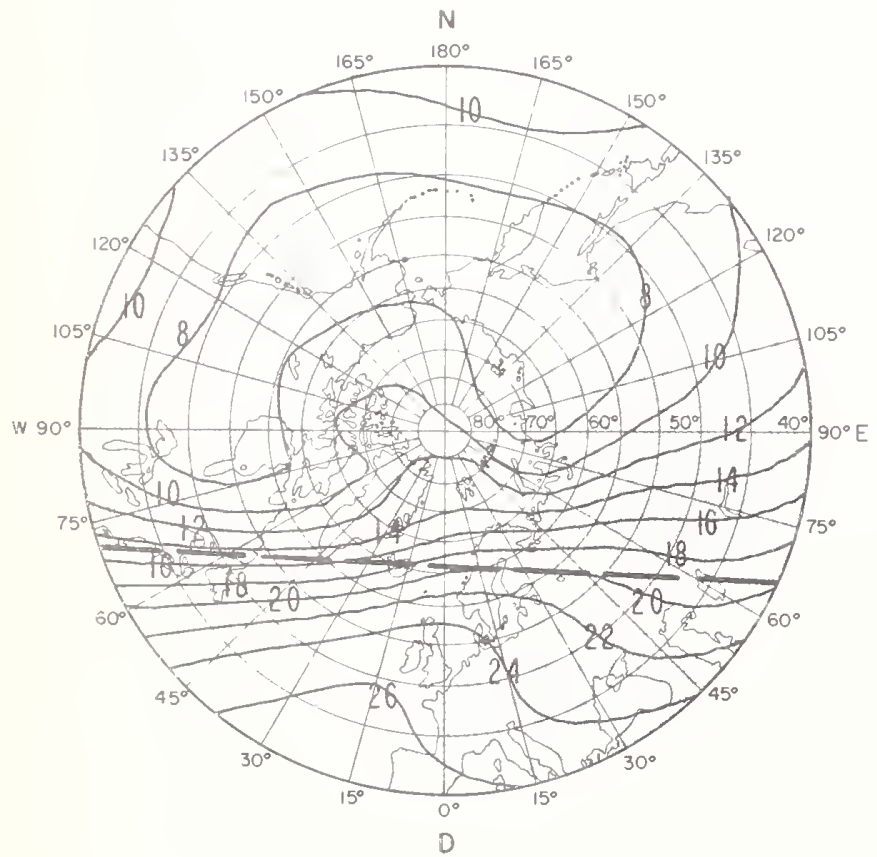

SOUTH POLAR AREA

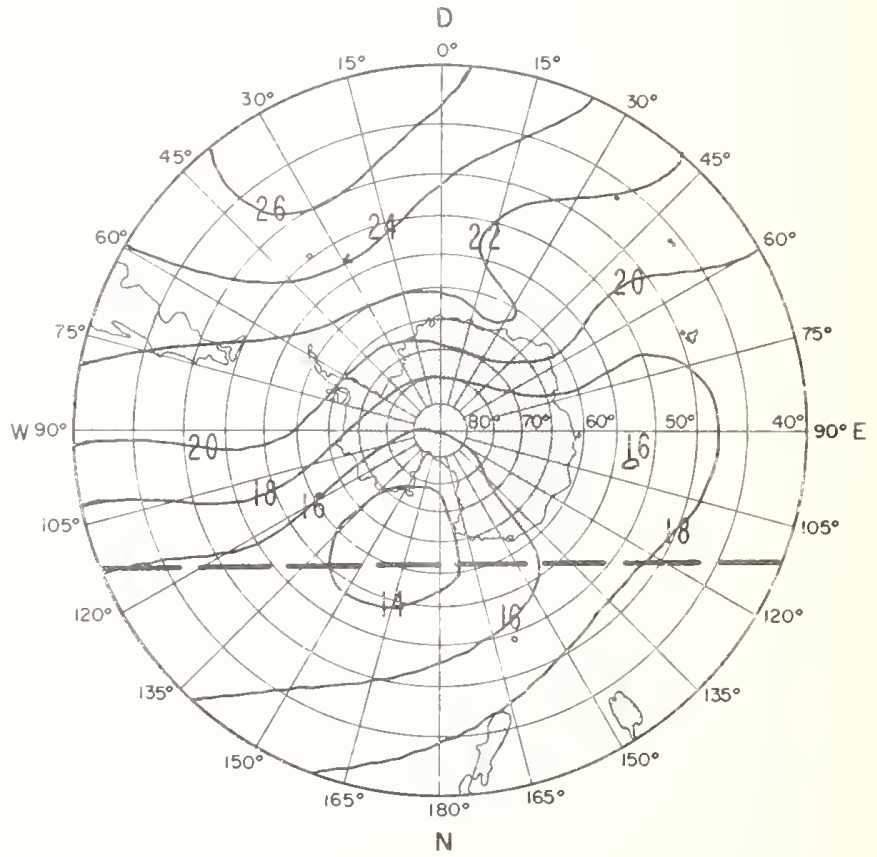



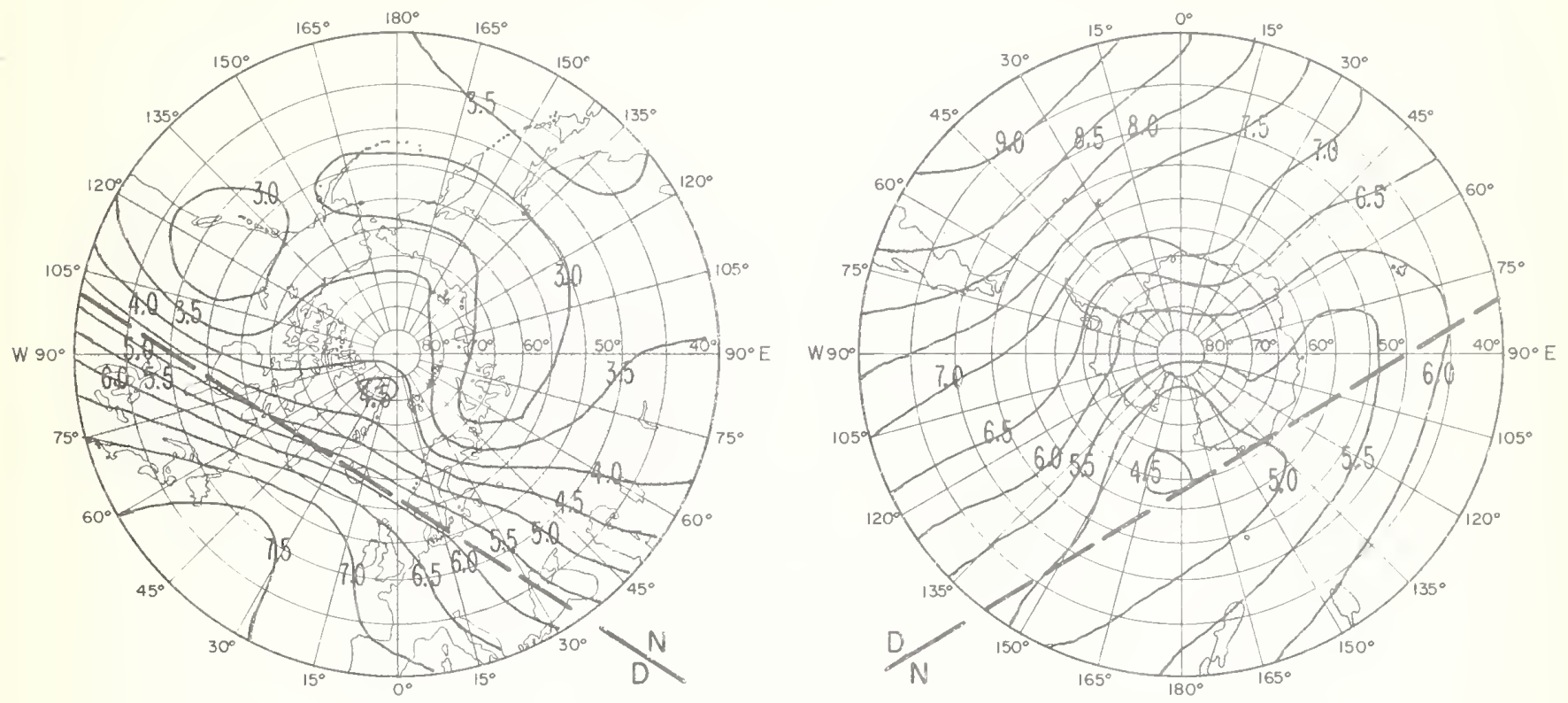

FIG.2OA. PREDICTED MEDIAN MUF(ZERO)F2 (MC/S)
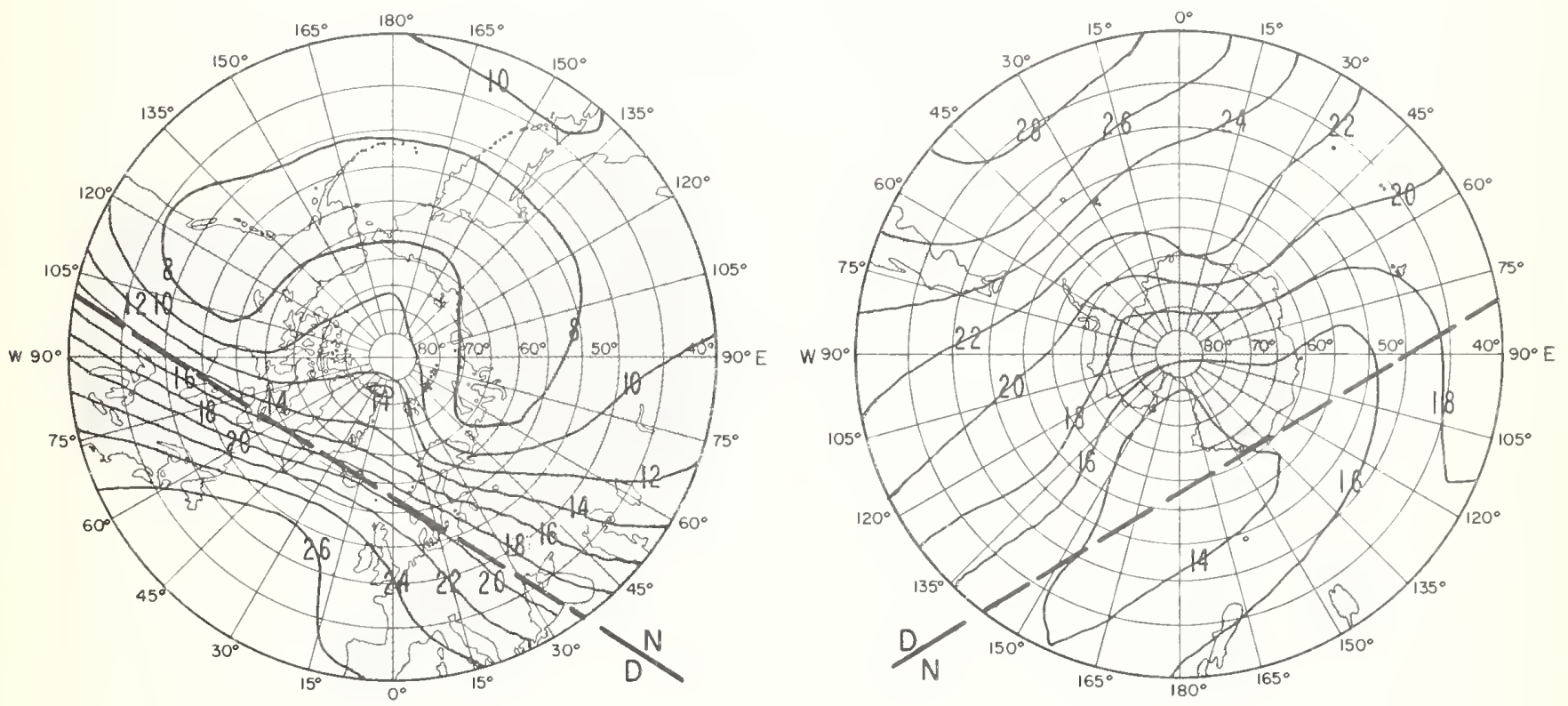

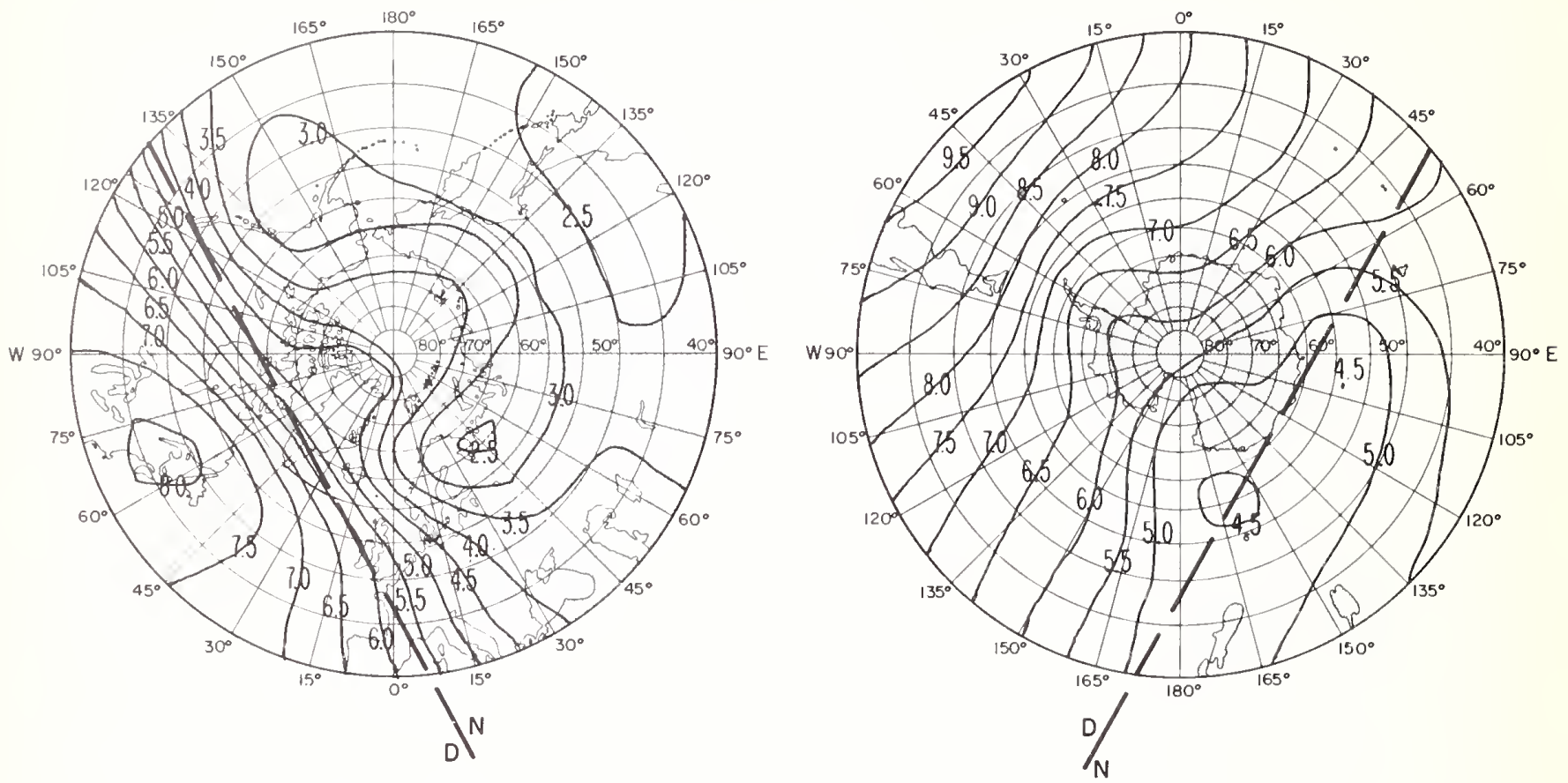

FIG.2 I A. PREDICTED MEDIAN MUF(ZERO)F2 (Mc/s)
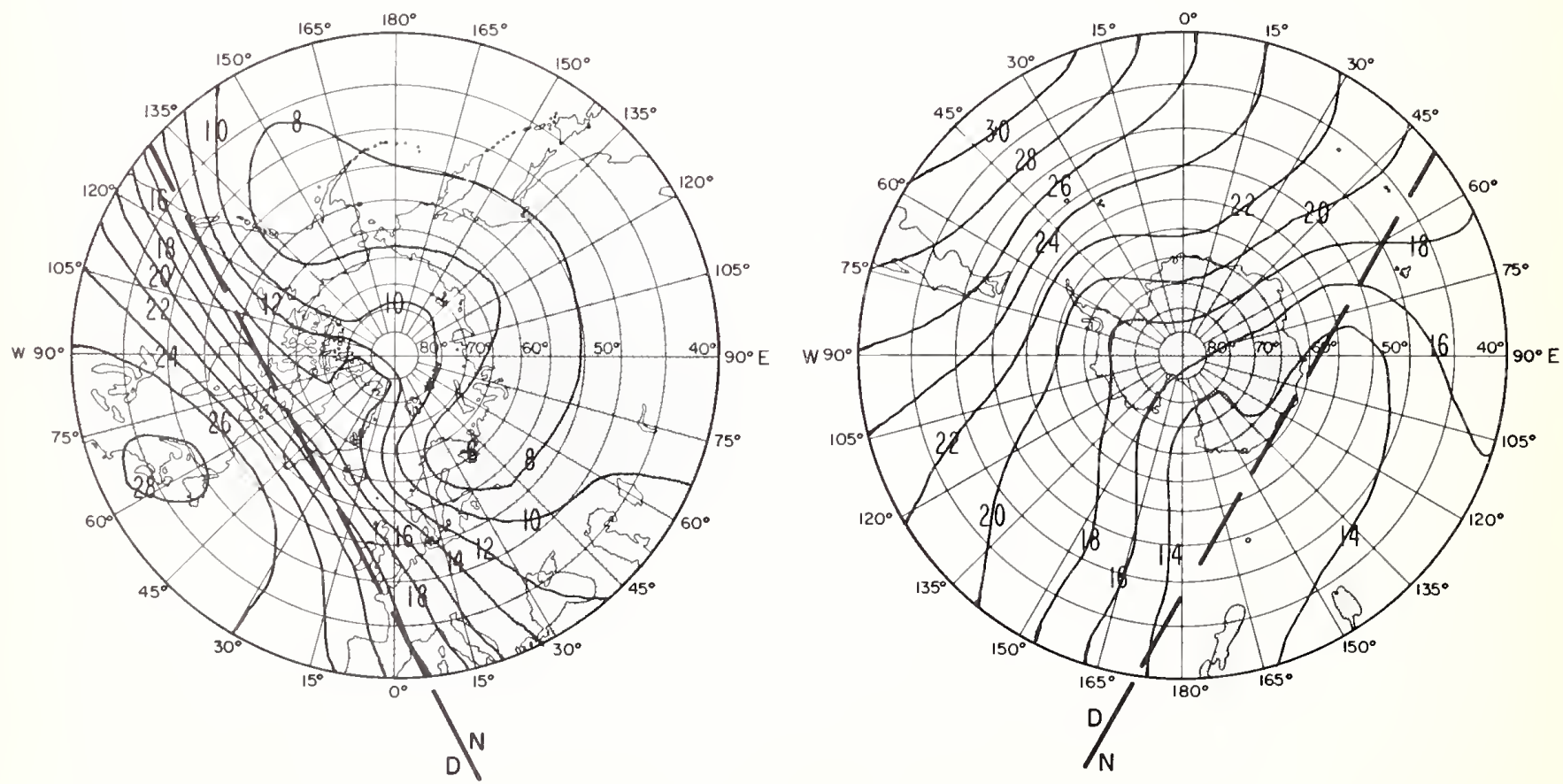

FIG. 2 I B. PREDICTED MEDIAN MUF (400O)F2 (MC/s) 

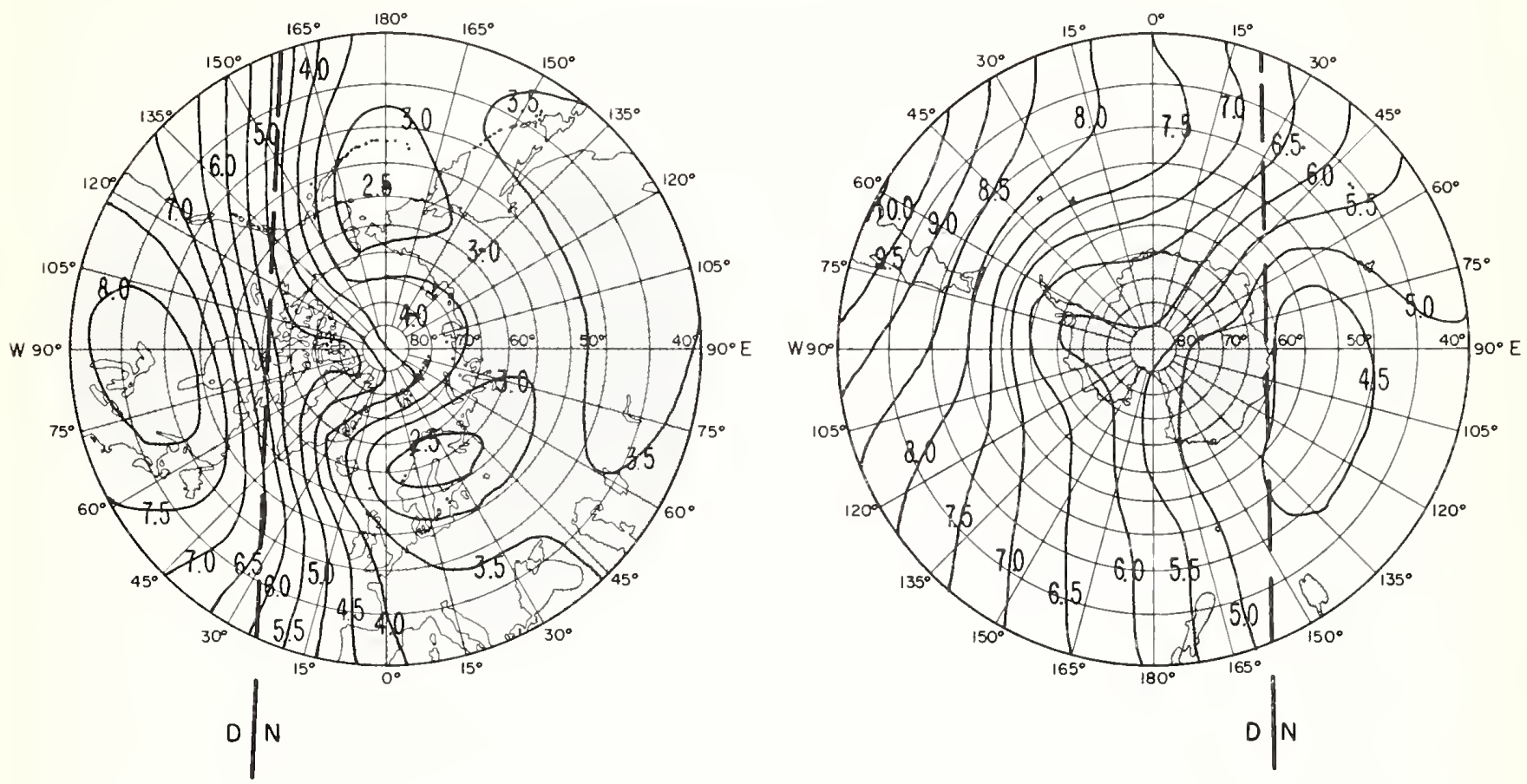

FIG. 22A. PREDICTED MEDIAN MUF(ZERO)F2 (MC/S)

NORTH POLAR AREA

SOUTH POLAR AREA
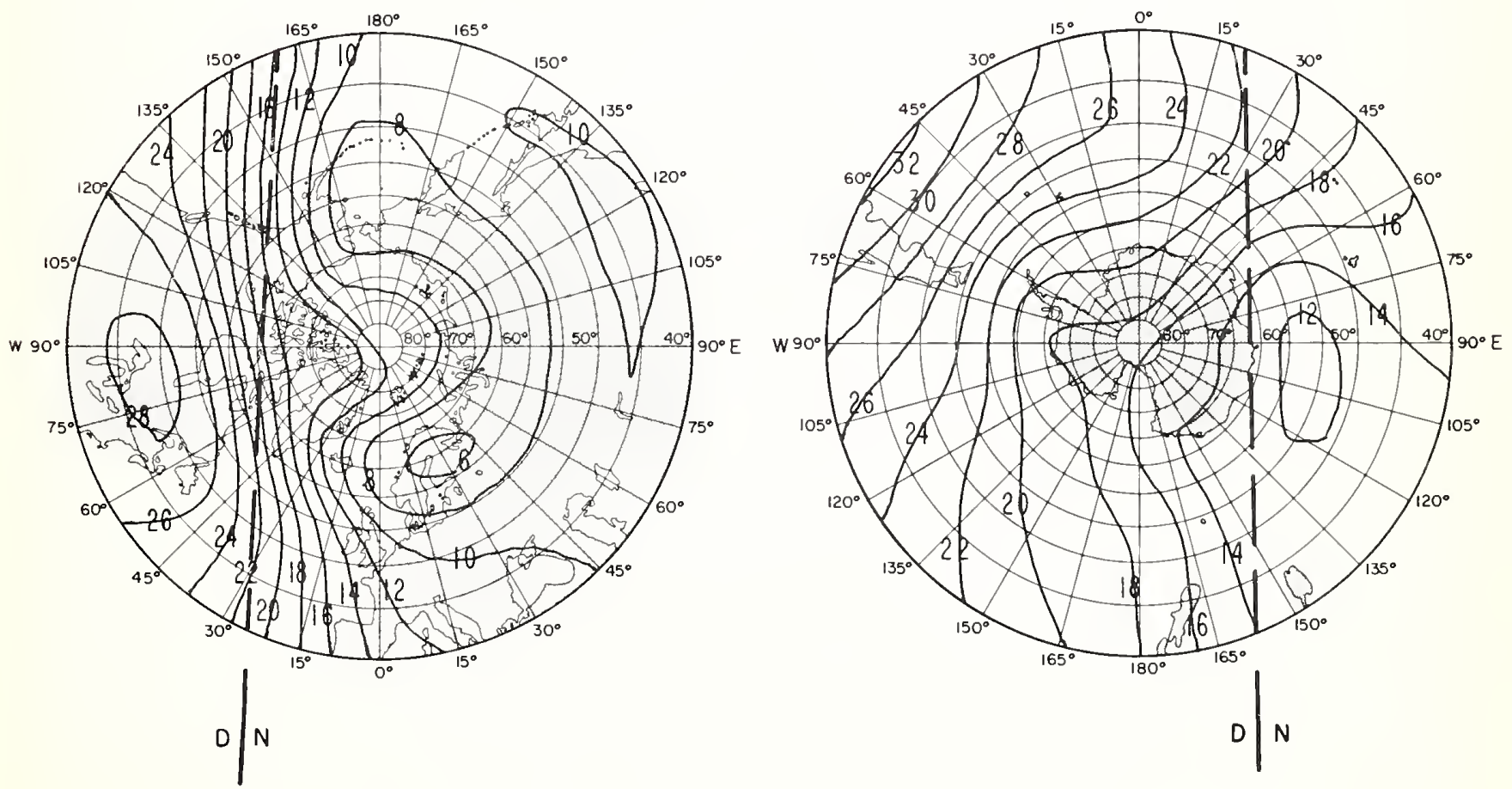

FIG. 2 2B. PREDICTED MEDIAN MUF (4000) F2 (MC/S) 

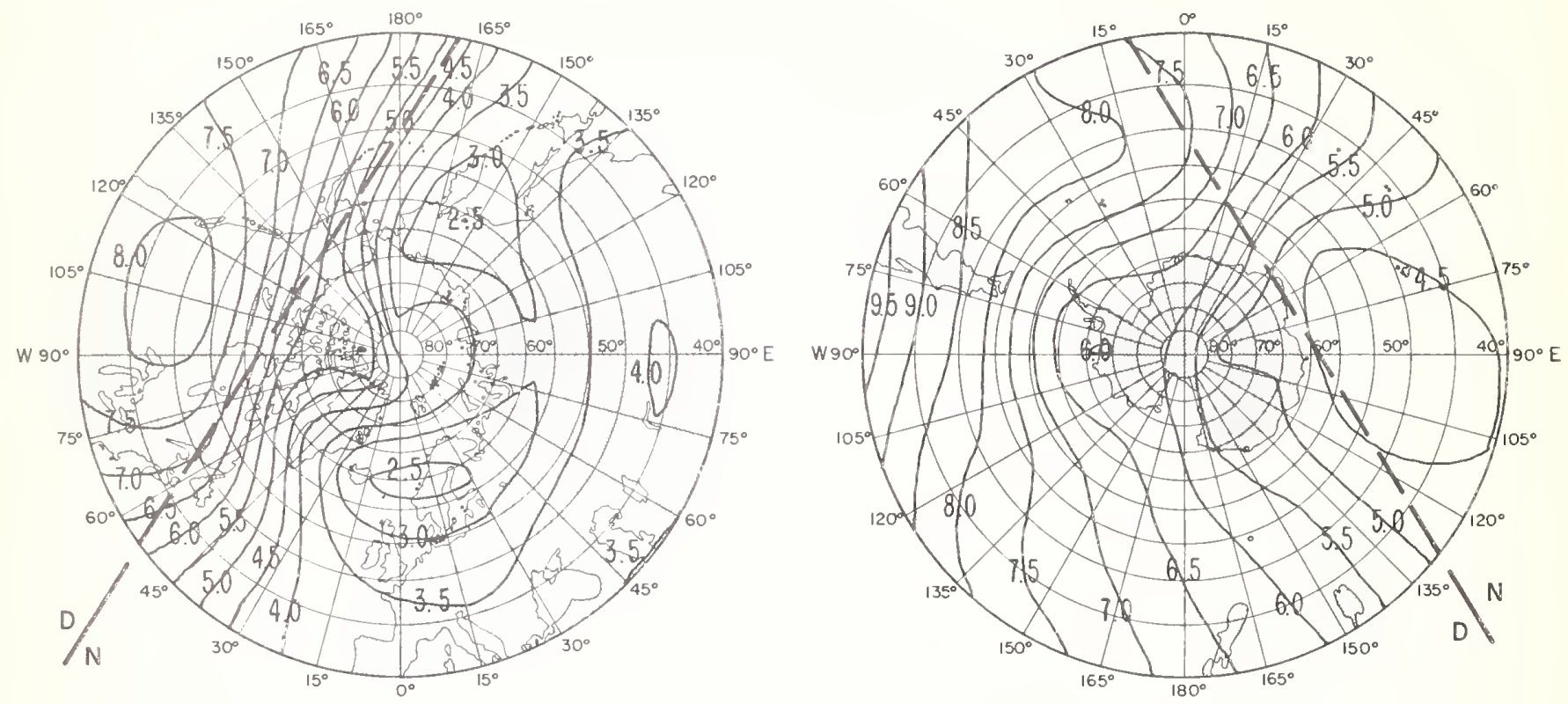

FIG.23A. PREDICTED MEDIAN MUF(ZERO)F2 (MC/S)
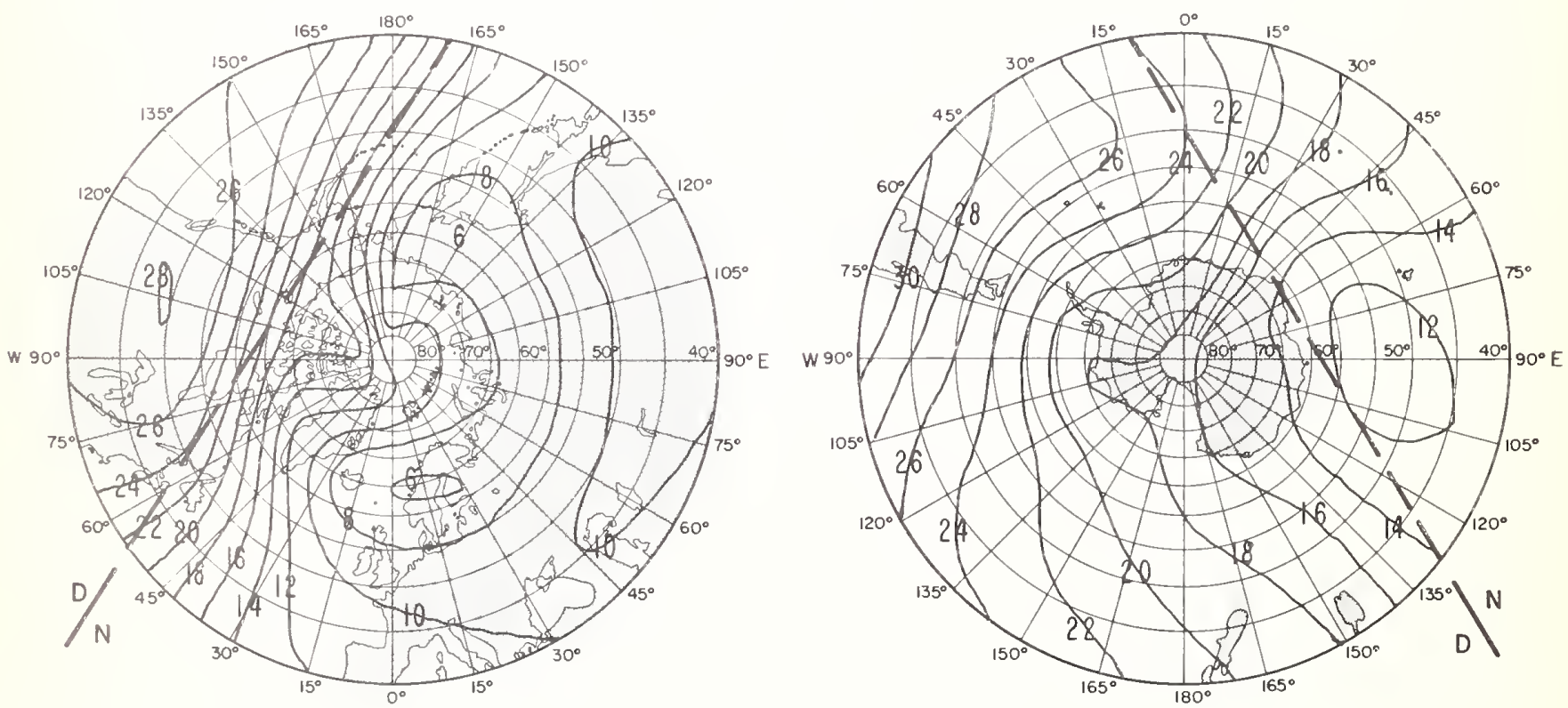

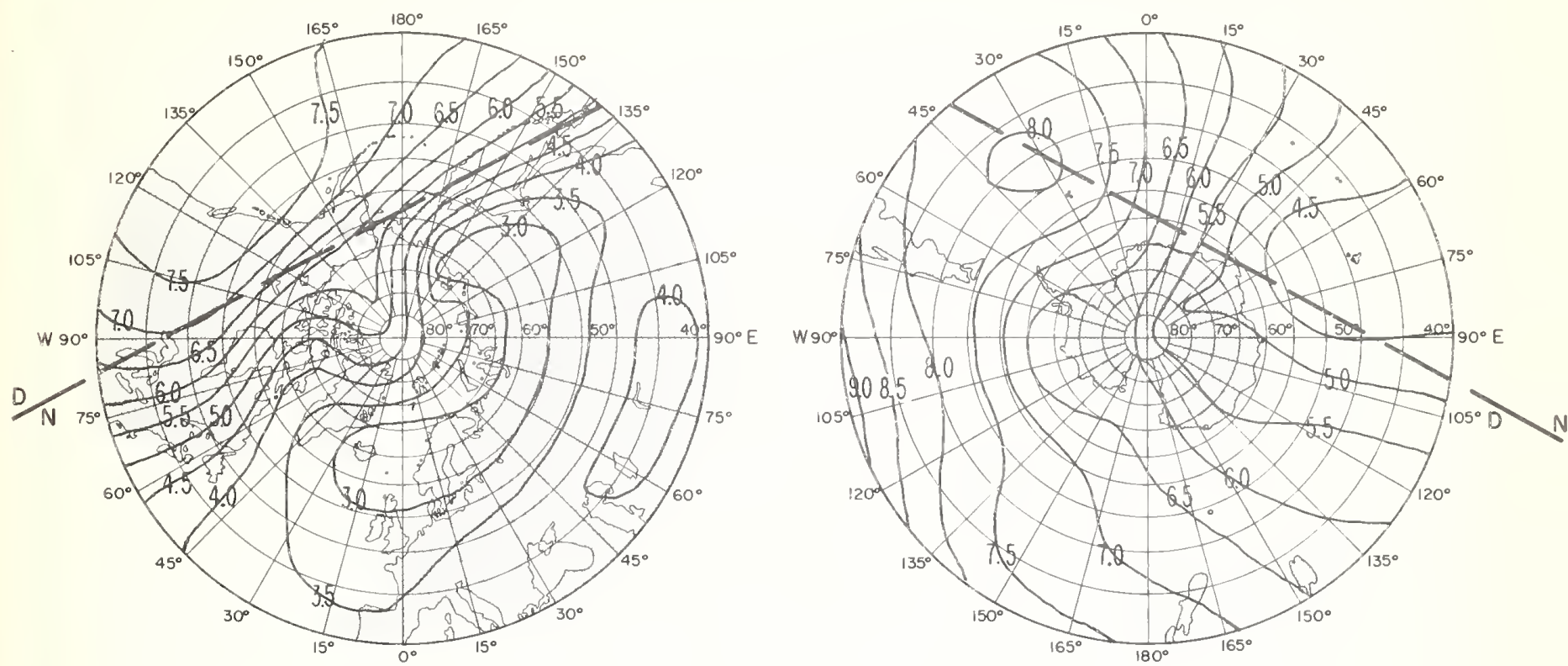

FIG. 24A. PREDICTED MEDIAN MUF(ZERO)F2 (MC/S)

NORTH POLAR AREA

SOUTH POLAR AREA
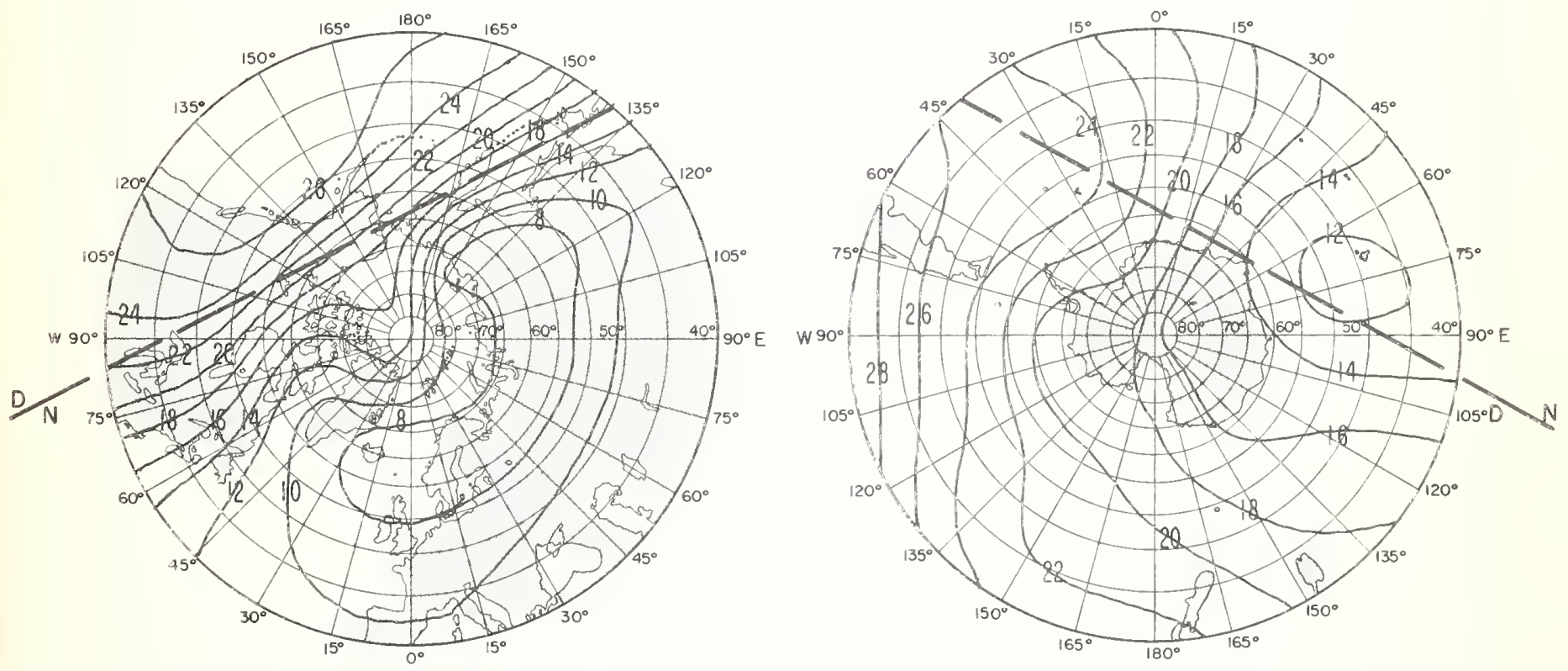

FIG. 24B. PREDICTED MEDIAN MUF (4000)F2 (MC/S) 
UNITED STATES

GOVERNMENT PRINTING OFFICE

DIVISION OF PUBLIC DOCUMENTS

WASHINGTON, D. C., 20402

OFFICIAL BUSINESS
POSTAGE AND FEES PAID

U.S. GOVERNMENT PRINTING OFFICE

DEPARTMENTS OF THE ARMY

AND THE AIR FORCE

Washington, D.C., 20301, 1 September 1965

TB 11-499-33/TO 31-3-28, Central Radio Propagation Laboratory Ionospheric Predictions for December 1965 , is published for the use of all concerned.

By Order of the Secretaries of the Army and the Air Force:

HAROLD K. JOHNSON, General, United States Army, Chief of Staff.

\section{OFFICIAL:}

J. C. LAMBERT,

Major General, United States Army.

The Adjutant General.

\section{OFFICIAL:}

\section{R. J. PUGH,}

Colonel, United States Air Force, Director of Administrative Services.

Distribution :

Active Army:

USASA (4); USA Elct Comd (5); USA MI Comd (2); USA Test \& Eval Comd (1); CC-E (1); US CONARC (3); ARADCOM (2); OS Maj Comd (5); OS Base Comd (2); Log Comd (2); MDW (1); Armies (5); Corps (2); Div (2); USAEPG (2); USA Elct RD Agcy, W (4); Svc Colleges (4); Br Svc Sch (4) except USASCS (20); USAADCEN (4); ARADCOM Rgn (2); WSMR (5); USA Elct RD Agcy A (4); JBUSMC (12); USA Corps (1); USA Mbl Spt Cen (1); USAMC (1); USACDA (1); USA Msl Spt Comd (1); Units org under fol TOE: 11-18 (1); 11-95 (1); 11-50 (AC) ; ATAD (1).

$N G$ : None.

USAR: None.

For explanation of abbreviations used, see AR 320-50. 